\title{
Adaptive Output Feedback Control for the Trajectory Tracking of High-Speed Trains with Disturbance Uncertainties on the Basis of Neural Network Observers
}

\author{
Yang Liu (i) and Weidong Li (i) \\ School of Electronic and Information Engineering, Dalian Jiaotong University, Dalian 116028, China \\ Correspondence should be addressed to Weidong Li; muxiaoyi123@sina.com
}

Received 15 January 2020; Revised 30 April 2020; Accepted 13 May 2020; Published 9 July 2020

Academic Editor: Paola Pellegrini

Copyright (C) 2020 Yang Liu and Weidong Li. This is an open access article distributed under the Creative Commons Attribution License, which permits unrestricted use, distribution, and reproduction in any medium, provided the original work is properly cited.

\begin{abstract}
The dynamic model of high-speed trains (HSTs) is nonlinear and uncertain; hence, with the decrease in the running interval of HSTs, an accurate and safe train operation control algorithm is required. In this study, an adaptive output feedback trajectory tracking control method for HSTs is proposed on the basis of neural network observers. The proposed method aims to solve problems, such as the immeasurable speed, model parameter disturbance, and unknown external disturbance of HSTs. In this method, a neural network adaptive observer is designed to estimate the velocity of an HST. Another neural network model is used to approximate the model uncertainties. Moreover, a robust controller is constructed by considering the train position and velocity tracking errors. In the proposed observer/controller, the bound function of estimator errors is introduced to increase the accuracy and safety of the tracking system. Furthermore, the adaptive update value of the neural networks, output weights, and bound function are performed online. All adaptive algorithms and the observer/controller are synthesized in nonlinear control systems. The error signals of the closed-loop trajectory tracking system are uniformly and eventually bounded through a formal proof on the basis of the Lyapunov methods. Simulation examples illustrate that the proposed controller is robust and has excellent tracking accuracy for system model parameter and external disturbance.
\end{abstract}

\section{Introduction}

Given their large capacity, low energy consumption, and low pollution, high-speed trains (HSTs) have become one of the most efficient modes in railway transportation, thereby attracting much attention toward the development of green transport [1]. However, the running process of HSTs is coupled with a complex system and a complex environment. In addition, such process is nonlinear, multiobjective, multiconstrained, and time varying. In summary, the dynamic model of HSTs is nonlinear and uncertain [2-4]. A primary task of automatic train operation (ATO) is speed regulation. A speed regulation control strategy based on a precise model cannot easily satisfy the requirements of safe and punctual operation. With the increase in train speed and the decrease in the running interval of HSTs, an accurate and reliable control strategy that uses an adaptive and intelligent method should be explored to guarantee a safe and comfortable operation.

Proportion integration differentiation (PID) control is widely used in train speed control to ensure the safety of train operation [5-7]. The PID control algorithm is simple and easy to implement and does not easily make mistakes. However, it easily causes the instability and energy consumption of train operation due to the excessive frequency of adjustment. Moreover, its parameter selection has no clear method. Thus, an optimal state of train operation control is difficult to achieve.

To solve the above problem, Sekine et al. [5] proposed a two-degree-of-freedom fuzzy neural network control method, which has a hierarchical structure of two sets of control knowledge. In [6,7], an adaptive fuzzy PID soft 
switching control method, which switches between the fuzzy and PID controllers, was first proposed for use in ATO systems. This method improved the smoothness, comfort, and parking accuracy of train operation. However, the above methods were based on the subway as research object or on ordinary electric multiple units with low running speed; such bases are unsuitable for HSTs because the nonlinear characteristics of the train model are evident in high-speed operation. For this reason, the authors in [8] used a datadriven modeling method to establish multiple local linear models to describe the dynamic characteristics of the HST running process. However, the basic contradiction of the linearization method in controlling the nonlinearly controlled object is difficult to avoid.

To enhance the processing of the nonlinear components of trains and achieve an improved accuracy for the speed tracking control of HSTs, the authors in [9] used the Elman neural network. The dynamic model of HSTs was established using substantial offline data, but the timevarying model and external disturbance in the process of train operation were not considered. Li et al. [10] divided the train system into linear and nonlinear components and used the least squares method and adaptive network-based fuzzy inference system (ANFIS) methods to model the linear and nonlinear components, respectively. However, the stability of the entire closed-loop system was not discussed because the controller was not designed in a unified framework.

In [11], a direct fuzzy controller was designed for onemass-point and multimass-point models, but the accurate model parameters are necessary and do not consider external disturbances. Combining $\mathrm{H}_{2}$ and $\mathrm{H}_{\infty}$ methods, the authors in [12] designed an HST cruise controller using accurate model parameters, thereby enhancing the robustness of the system. However, the stability analysis of the closed-loop system was based on linearization theory. Thus, the overshoot of tracking error was relatively large. In consideration of the nonlinear characteristic and uncertain parameters of HSTs, a model predictive controlmixed $\mathrm{H}_{\infty}$ algorithm was designed in [13], thereby achieving velocity tracking control in the case of model disturbance. However, only the matching uncertainties were considered. In [14], a sliding mode adaptive robust controller for HTS was designed on the basis of Lyapunov stability theory. However, no adaptive estimation or robust terms were available for uncertain model parameters and external disturbance. As a result, the convergence of a closed-loop system depended on the design parameters, thus leading to excessive steady-state errors. In [15], a robust adaptive controller based on Lyapunov stability analysis was designed for a multimass-point model and considered external disturbance and parameter uncertainty. In the case of HSTs' repeated running on tracks, an iterative algorithm was studied in the tracking control [16-19]. However, iterative learning required the motion of HST to be repetitive; that is, the reference speed curve of the train must be unchanged. Thus, the real-time performance of the iterative method was poor, and its convergence speed must be further improved [20].
With the increase in the speed of HST and the development of intelligent transportation, moving block technology must be introduced to improve operational efficiency. Thus, an optimized safety tracking distance is necessary. In this case, the following must be considered: the strong nonlinearity and the uncertainty of the dynamic model of HSTs, the uncertain mismatching under strong wind conditions, the robustness of the controller, and the accuracy of the closed-loop system.

The combination of nonlinear and intelligent methods is a good solution to the above problems. In [21], a trajectory tracking controller of wheeled mobile manipulators was designed on the basis of the fuzzy neural network and extended Kalman filtering; as a result, the tracking problem of wheeled robot under uncertainty is solved. In [22], an adaptive neural network controller of a fully actuated marine surface vessel was proposed. Based on above, the neural network and integral sliding mode method were combined by [23] to design a tracking controller for fully driven surface ships.

In $[24,25]$, the sliding mode and neural network, the back stepping, and the fuzzy algorithm were used to design a tracking controller for aircraft. In [26], an adaptive sliding mode control system with a double-loop recurrent neural network structure was proposed and applied to the motion control of a gyroscope. In [27], an adaptive high-gain observer was used to estimate the unmeasured state for a nonlinear second-order system with external disturbance. In $[28,29]$, an adaptive fuzzy observer was designed on the basis of stability theory. In [30,31], a neural network observer was designed for an uncertain strict feedback system and a nonlinear stochastic system, respectively. In [32], the output feedback control of wheeled mobile robot formation was designed by combining neural network observers. In $[33,34]$, neural network observers were used to design a trajectory tracking controller for underactuated vehicles. In [35], a neural network observer was designed for noninteger order systems.

Researchers have exerted much effort in identifying and determining the value of a model parameter. The resistance of the HST is related to its operating environment, and the resistance parameter of different HST types also varies. Moreover, obtaining an accurate parameter is difficult, and the train speed variation has a strong nonlinear relationship with the resistance it receives. In addition, the sensor measurement error or sensor failure hinders train operation safety.

These factors restrict the increase in train speed. Thus, we focus on the following: nonlinear characteristics of the HST model, time-variable model parameters, unknown disturbance of the external environment, and immeasurable HST speed. Motivated by these observations, this study proposes an adaptive neural observer- (ANO-) based output feedback controller for the trajectory tracking control of HSTs. The major contributions of this study can be summarized as follows:

(1) References [2-12] only dealt with the speed tracking problem of HSTs. In consideration of the demand for multitrain tracking control of the moving block and 
in combination with the RBF adaptive and sliding mode methods, a displacement and a speed tracking control strategy for HSTs are designed. The sliding mode method is used to ensure the stability of the tracking control. An adaptive neural network is used to estimate the nonlinearity and uncertainty of the HST to ensure the accuracy of the tracking control. A bounded estimation function of design error is used to enhance the robustness of the system.

(2) The tracking control algorithm does not require the running speed of an HST. On the basis of [21-32], an adaptive neural network observer is designed under the condition of unknown dynamic model parameters and external interference of HST. Thus, the HST equipment and adaptive neural observer can be reconstructed into pure feedback form.

(3) The design parameters of the proposed observer/ controller are adjusted online, and the stability and convergence of the closed-loop system are proven through a formal proof based on the Lyapunov methods.

This paper is organized as follows. Section 2 introduces the problem formulation. An ANO is designed in Section 3. Section 4 presents the proposed controller. In Section 5, an analysis of stability theories is discussed. In Section 6, the main result for trajectory tracking is presented in the form of a theorem, and a rigorous mathematical explanation is provided. Conclusions are presented in Section 7.

\section{Problem Formulation}

With only the longitudinal motion of the train considered, the dynamic model of an HST can be expressed as follows [36]:

$$
\left\{\begin{array}{l}
\frac{\mathrm{d} s}{\mathrm{~d} t}=\dot{s}(t)=v, \\
M \frac{\mathrm{d} v}{\mathrm{~d} t}=M \dot{v}(t)=F, \\
F=u(t)-f_{d}(v, s), \\
f_{d}(v, s)=f_{a}(s)+f_{b}(v),
\end{array}\right.
$$

where $t$ represents a continuous time indicator; $s(t)(\mathrm{m})$ and $v(t)(\mathrm{m} / \mathrm{s})$ are the operating displacement and speed of the train, respectively; $M>0$ is the equivalent total mass of the train system, including the weights of the train, passenger, and baggage; $F(N)$ is the resultant force of the train; $u(t)(\mathrm{N})$ is the control input of the train, that is, the traction or braking force of the train; $f_{d}(v, s)(\mathrm{N})$ represents the total resistance to the train operation; $f_{a}(s)(\mathrm{N})$ is the additional resistance to the train operation; and $f_{b}(v)(\mathrm{N})$ is the general resistance of the train operation.

$f_{a}(s)$ is a resistance other than the general resistance, which mainly includes slope additional resistance (as shown in Figure 1), curve resistance, and tunnel resistance.where $L_{s}$ is the tunnel length.

(1) Slope additional resistance: as shown in Figure 1, the slope additional resistance force $f_{s}$ comes from the grade of the track at the point of the train's location and is modeled as

$$
f_{s}(t)=M(t) g \sin \alpha(t)
$$

where $\alpha(t)$ is the slope angle of the current track. The track line comprises several horizontal, slope, and curvature tracks. For a certain slope track, the slope angle $\theta$ is a constant.

(2) Curve resistance: the curvature force $f_{c}$, which comes from the curvature of the track at the point of the train's location, is modeled as

$$
f_{c}(t)=0.1029 \frac{\varphi(t)}{L_{r}(t)} M(t)
$$

where $\varphi(t)$ is the center angle of the curve and $L_{r}(t)$ is the curve length.

(3) Tunnel resistance:

$$
f_{t}(v, t)= \begin{cases}\frac{v^{2}}{10^{9}} L_{s} M(t), & \text { exist restricted ramps in the tunnel, } \\ \frac{1.3}{10^{6}} L_{s} M(t), & \text { no restricted ramp in the tunnel, }\end{cases}
$$

$f_{a}$ is obtained as follows:

$$
f_{a}(v, t)=f_{s}(t)+f_{c}(t)+f_{t}(v, t)
$$

The curve or tunnel can also be converted into an equivalent ramp to obtain the slope angle. Equation (5) can be changed as

$$
f_{a}(s)=l(t) \sin (r(s))
$$

where $l(t)$ is the time-varying additional resistance parameter and $r(s)$ is the time-varying equivalent slope angle of the train displacement. Given that the displacement is the integral of the velocity, equation (6) can be changed to

$$
f_{a}(v, t)=l(t) \sin \left(r\left(\int_{t_{1}}^{t_{2}} v(t)\right)\right),
$$

where $t_{1}$ represents the start time of the additional resistance of the train and $t_{2}$ represents the end time of the additional resistance of the train.

General resistance consists of mechanical and air resistance and is expressed as

$$
f_{b}(v, t)=a_{0}(t)+a_{1}(t) v+a_{2}(t) v^{2},
$$

where $a_{0}(t), a_{1}(t)$, and $a_{2}(t)$ are the general resistance parameters of the train with time variation. 


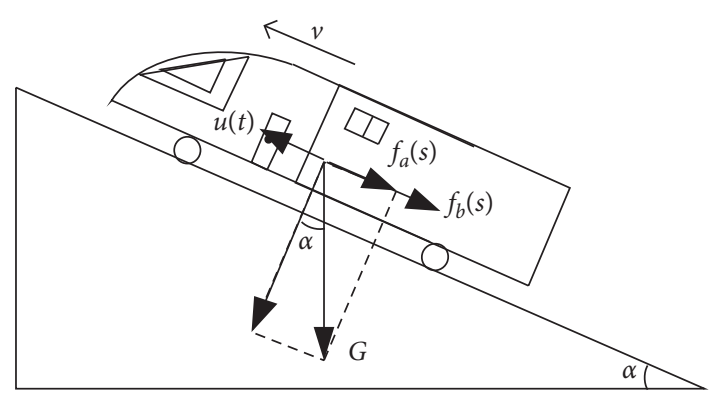

Figure 1: Diagram of slope additional resistance.

Therefore, the HST motion model adopted in this study can be written as follows:

$$
\left\{\begin{array}{l}
\dot{s}(t)=v \\
\dot{v}(t)=\frac{\chi}{M} u(t)-\frac{\chi}{M} f_{d}(v, t)+d(t) \\
f_{d}(v, t)=a_{0}(t)+a_{1}(t) v+a_{2}(t) v^{2}+l(t) \sin \left(r \int_{t_{1}}^{t_{2}} v(t)\right)
\end{array}\right.
$$

where $d(t)$ represents unmolded dynamics, such as external disturbance.

The common system assumptions in nonlinear and neural network controls are provided to discuss the convergence of the algorithm.

Assumption 1. The reference trajectory $x_{d}=\left[s_{d}, v_{d}\right]$ is smooth. The first and second derivatives of $x_{d}$ exist and are bounded.

Assumption 2. For the given desired trajectory $x_{d}(t)=$ $\left[s_{d}, v_{d}\right] \in C^{2}[0, T]$, a suitable traction/brake control input $u(t)$ can drive the train displacement and velocity $x=[s, v]$. Thus, the desired trajectory $x_{d}$ over a fixed finite time interval $t \in[0, T]$, and $\dot{s}_{d}=v_{d}$ is accurately tracked.

Assumption 3. Train parameters $M, a_{0}(t), a_{1}(t)$, and $a_{2}(t)$ are unknown but continuously bounded, and they are greater than zero. The time-varying additional resistance parameter $l(t)$ and external disturbance $d(t)$ are unknown and continuously bounded.

Assumption 4. The ideal NN weights are bounded such that $\left\|W^{*}\right\| \leq\left\|W_{N}\right\|$, and $\left\|W_{N}\right\|$ is the boundary of $\left\|W^{*}\right\|$.

Assumption 5. The nonlinear term $f_{d}$ in equation (9) satisfies the following Lipchitz condition:

$$
\left\|f_{d}\left(v_{1}\right)-f_{d}\left(v_{2}\right)\right\| \leq L_{1}\left\|v_{1}-v_{2}\right\|+L_{1}\left\|v_{1}-v_{2}\right\|^{2},
$$

where $L_{i} \in \mathfrak{R}^{+}=1,2$ is unknown and constant.

The control objective is that, for the mathematical model (9) of HST and under Assumptions 1-3, the parameters and disturbances of the HST model are unknown according to the train position information. The output feedback control law is designed to make the HST run along the expected trajectory and ensure that the closed-loop signal is consistent and ultimately bounded to achieve HST tracking.

\section{Adaptive Neural Network Observer Design}

The position of a train can be given by GPS and other equipment. Meanwhile, the speed information of the train is measured using a speed sensor, which may have measurement errors or sensor failure. Therefore, this section focuses on estimating the speed information in accordance with the position information of the HST.

First, the observation error is defined:

$$
\left\{\begin{array}{l}
\widetilde{s}=\widehat{s}-s, \\
\widetilde{v}=\widehat{v}-v,
\end{array}\right.
$$

where $\widehat{s}$ is the estimation of position; $\widehat{v}$ is the estimation of speed; $\widetilde{s}$ and $\widetilde{v}$ are the estimation errors of $s$ and $v$, respectively.

The adaptive update laws of the observer are designed as follows:

$$
\left\{\begin{array}{l}
\dot{\vec{s}}=\widehat{v}-k_{1} \widetilde{s} \\
\dot{\hat{v}}=\widehat{f}-k_{2} \widetilde{s}-k_{3} \dot{\vec{s}}+D_{1} \\
f=\frac{\chi}{M} u(t)-\frac{\chi}{M} f_{d}(v, t)+d(t)
\end{array}\right.
$$

where $\hat{f}$ is the estimate of $f, D_{1}$ is a robust term whose specific expression is given in the subsequent analysis, and $k_{1}$ is positive and a design parameter.

The time derivative of equation (11) is determined and integrated in equations (9) and (12) to obtain the following:

$$
\begin{aligned}
& \dot{\tilde{s}}=\widetilde{v}-k_{1} \widetilde{s}, \\
& \dot{\tilde{v}}=\widehat{f}\left(z_{1}\right)-f\left(z_{1}\right)-k_{2} \widetilde{s}-k_{3} \dot{\tilde{s}}+D_{1} .
\end{aligned}
$$

The parameters $a_{0}(t), a_{1}(t), a_{2}(t)$, and $l(t)$ in equation (12) are based on Assumption 3 and are continuously bounded variables. Thus, the function $f\left(z_{1}\right): \Omega \longrightarrow \mathfrak{R}$ is bounded and can be approximated using the neutral network given by the following:

$$
f=W_{f}^{* T} h_{f}\left(z_{1}\right)+\varepsilon_{f}\left(z_{1}\right), \quad\left|\varepsilon_{f}\left(z_{1}\right)\right| \leq \varepsilon_{f, N},
$$

where $z_{1}=[s, v, u]$ is the input of the neural network; $W_{f}^{*} \in \mathfrak{R}^{m},\left\|W_{f}^{*}\right\| \leq\left\|W_{f, N}\right\|$, and $\left\|W_{f, N}\right\|$ are the boundaries of $\left\|W_{f}^{*}\right\| ; m$ is the number of neurons in the hidden layer; $\varepsilon_{f}(z)$ is the neural network reconstruction error; $\varepsilon_{f, N}$ is the boundary value of $\varepsilon_{f}(z)$; and $h_{f}\left(z_{1}\right)=\left[h_{f, 1}, h_{f, 2}, \ldots\right.$, $\left.h_{f, m}\right]^{\mathrm{T}}: \mathfrak{R}^{3} \longrightarrow \mathfrak{R}^{m}$ is the basic function vector. The basic function $h_{f}\left(z_{1}\right)$ is selected as

$$
h_{f, j}=\exp \left(\frac{-\left\|z_{1}^{T}-c_{f, j}\right\|^{2}}{\sigma_{f, j}^{2}}\right),
$$

where $c_{f, j}=\left[c_{f, j, 1}, c_{f, j, 2}, c_{f, j, 3}\right] \in \mathfrak{R}^{3}$ is the center value of the Gaussian function, $\sigma_{f, j}$ is the ith hidden layer node, and $j=1,2, \ldots, m$. 
Moreover, the velocity vector is unknown. Therefore, the following neural network is used for estimation:

$$
\widehat{f}=\widehat{W}_{f}^{T} h_{f}\left(\widehat{z}_{1}\right) \text {, }
$$

where $\widehat{z}_{1}=[\widehat{s}, t \widehat{v} n, q u]$ is the input of the neural network and $\widehat{W}_{f}$ represents the estimated value of $W_{f}^{*}$ used to observe $\widehat{v}$ in equation (12).

Substituting equations (13), (15), and (16)into equation (14), we obtain the closed-loop error dynamic equations as

$$
\dot{\tilde{v}}=-k_{3} \widetilde{v}-\left(k_{2}-k_{1} k_{3}\right) \widetilde{s}+\widetilde{W}_{f}^{T} h_{f}\left(\widehat{z}_{1}\right)+D_{1}+\widetilde{\varepsilon}_{f}\left(z_{1}\right),
$$

where the estimation error $\widetilde{W}_{f}=\widehat{W}_{f}-W_{f}^{*}, \widetilde{\varepsilon}_{f}\left(\widetilde{z}_{1}\right)=$ $W_{f}^{* T}\left(h_{f}\left(\widehat{z}_{1}\right)-h_{f}\left(z_{1}\right)\right)-\varepsilon_{f}\left(z_{1}\right)$. According to the inequality $\left\|h_{f}\left(\widehat{z}_{1}\right)-h_{f}\left(z_{1}\right)\right\| \leq L_{\phi}\left\|\widehat{z}_{1}-z_{1}\right\|$ [34], the neutral network weights following the updating law are as follows:

$$
\dot{\hat{W}}_{f}=\Gamma_{f}\left(-h_{f}\left(\widehat{z}_{1}\right)\left(\dot{\tilde{s}}+t k_{1} n \widetilde{s}\right)+\sigma_{f}\left(\widehat{W}_{f}-W_{f 0}\right)\right) \text {, }
$$

where $\Gamma_{f} \in \mathfrak{R}^{m \times m}$ and is positive; $\sigma_{f}$ is the design parameter and is positive; and $W_{f 0}$ is the $W_{f}^{*}$ a priori estimate.

A bounded function $\delta=\widetilde{\mathcal{\varepsilon}}_{f}\left(z_{1}\right)$ exists for $\widetilde{\mathcal{\varepsilon}}_{f}(\widetilde{z})$, and $\widehat{\delta}$ is the estimation of $\delta$. The updating law of $\widehat{\delta}$ is designed, and the robust term $D_{1}$ is defined as follows:

$$
D_{1}=-\gamma\left(\dot{\tilde{\mathcal{S}}}+t k_{1} n \widetilde{\boldsymbol{s}}\right) \widehat{\delta} .
$$

The adaptive updating law is designed as follows:

$$
\dot{\widehat{\delta}}=\alpha_{1}\left(\gamma\left(\dot{\tilde{s}}+t k_{1} n \widetilde{s}\right)\left(\dot{\tilde{s}}+t k_{1} n \widetilde{\mathcal{S}}\right)+\beta_{1}\left(\widehat{\delta}-t \delta_{0}\right)\right),
$$

where $\gamma\left(\dot{\tilde{s}}+t k_{1} n \widetilde{s}\right)=\tanh \left(\left(\dot{\widetilde{s}}+t k_{1} n \widetilde{s}\right) / \kappa_{1}\right)$ and $\kappa_{1}>0$ is a design constant.
Remark 1. $\gamma$ is a smooth function and satisfies $\left|\dot{\widetilde{s}}+t k_{1} n \widetilde{s}\right| \leq\left(\dot{\widetilde{s}}+t k_{1} n \widetilde{s}\right) \gamma\left(\dot{\widetilde{s}}+t k_{1} n \widetilde{s}\right)+\kappa_{1} \quad$ [37]. In addition, the function $\gamma$ can also be selected as $\gamma\left(\dot{\vec{s}}+t k_{1} n\right.$ $\widetilde{s})=\left(\dot{\widetilde{s}}+t k_{1} n \widetilde{s}\right) / 4 \kappa_{1}$.

Remark 2. If we select the estimated adaptive updating law as equations (12), (19), and (21), then the estimation error $(\widetilde{s}, t \widetilde{v})$ can be set to approximately zero.

\section{Output Feedback Controller Design}

In this section, the sliding mode method is introduced to design the tracking controller of an HST to reference displacement and speed.

The output value of the designed speed observer is used because the speed is immeasurable. Therefore, the displacement tracking error and the speed tracking error are obtained as follows:

$$
\left\{\begin{array}{l}
e_{s}=s_{d}-\widehat{s} \\
\dot{e}_{s}=\dot{s}_{d}-\widehat{v}
\end{array}\right.
$$

The filter error is designed as follows:

$$
e_{v}=k_{4} e_{s}+\dot{e}_{s}
$$

The time derivative of equation (21) can be obtained as

$$
\dot{e}_{v}=k_{4}\left(\dot{s}_{d}-\dot{\widehat{s}}\right)+\left(\ddot{s}_{d}-\dot{\widehat{v}}\right)
$$

where $k_{4}$ is a positive designed parameter.

Equation (24) by $M / \chi$ is used, and (12) is substituted into (24) to obtain the following:

$$
\begin{aligned}
\frac{M}{\chi} \dot{e}_{v}= & \frac{k_{4} M}{\chi}\left(\dot{s}_{d}-\left(\widehat{v}-k_{1} \widetilde{s}\right)\right)+\frac{M}{\chi} \ddot{s}_{d} \\
& -\frac{M}{\chi}\left(\hat{f}-f-\frac{\chi}{M} f_{d}(v)+d(t)-k_{2} \widetilde{s}-k_{3} \dot{\tilde{s}}+D_{1}\right)-u(t) .
\end{aligned}
$$

Considering $\quad D_{1}=-\gamma\left(\dot{\tilde{s}}+t k_{1} n \widetilde{s}\right) \widehat{\delta}, \quad f=W_{f}^{* T} h_{f}\left(z_{1}\right)+$ $\varepsilon_{f}\left(z_{1}\right), \widehat{f}=\widehat{W}_{f}^{T} h_{f}\left(\widehat{z}_{1}\right)$, and $\tilde{W}_{f}=\widehat{W}_{f}-W_{f}^{*}$, we can write equation (25) as follows:

$$
\begin{aligned}
\frac{M}{\chi} \dot{e}_{v}= & \frac{k_{4} M}{\chi}\left(\dot{s}_{d}-\widehat{v}+k_{1} \widetilde{s}\right)-\frac{M}{\chi} d(t)-u(t) \\
& -\frac{M}{\chi}\left(-\ddot{s}_{d}+\tilde{W}_{f}^{\mathrm{T}} h_{f}\left(\widehat{z}_{1}\right)+\widetilde{\varepsilon}_{f}\left(z_{1}\right)-\frac{\chi}{M} f_{d}(v)-k_{2} \widetilde{s}-k_{3} \dot{\tilde{s}}-\gamma\left(\dot{\vec{s}}+k_{1} \widetilde{s}\right) \widehat{\delta}\right) \\
= & f_{v}-\frac{M}{\chi} d(t)-u(t),
\end{aligned}
$$


where

$$
\begin{aligned}
f_{v}= & \frac{k_{4} M}{\chi}\left(\dot{s}_{d}-\widehat{v}+k_{1} \widetilde{s}\right) \\
& -\frac{M}{\chi}\left(\widetilde{W}_{f}^{T} h_{f}\left(\widehat{z}_{1}\right)+\widetilde{\varepsilon}_{f}\left(z_{1}\right)-\frac{\chi}{M} f_{d}(v)-k_{2} \widetilde{s}-k_{3} \dot{\tilde{s}}-\gamma\left(\dot{\tilde{s}}+k_{1} \widetilde{s}\right) \widehat{\delta}-\ddot{s}_{d}\right) .
\end{aligned}
$$

According to Assumptions 2 and 3, the nonlinear function $f_{v}$ is unknown and bounded. Thus, the RBF neural network is introduced to approximate $f_{v}$ as follows:

$$
f_{v}=W_{v}^{* T} h_{v}\left(z_{2}\right)+\varepsilon_{v}\left(z_{2}\right), \quad\left|\varepsilon_{v}\left(z_{2}\right)\right| \leq \varepsilon_{v, N},
$$

where $z_{2}=\left[s, \dot{s}, \widehat{v}, s_{d}, \dot{s}_{d}, \ddot{s}_{d}\right] \in \mathfrak{R}^{6}$ is the input vector of the neural network; $W_{v}^{*} \in \mathfrak{R}^{q},\left\|W_{v}^{*}\right\| \leq\left\|W_{v, N}\right\|$, and $\left\|W_{v, N}\right\|$ are the boundaries of $\left\|W_{v}^{*}\right\| ; q$ is the number of neurons in the hidden layer; $\varepsilon_{v}\left(z_{2}\right)$ is the neural network's forced reconstruction error and $\varepsilon_{v, N}$ is the boundary value of $\varepsilon_{v}\left(z_{2}\right)$; and $h_{v}\left(z_{2}\right)=\left[h_{v, 1}, h_{v, 2}, \ldots, h_{v, q}\right]^{T}: \mathfrak{R}^{6} \longrightarrow \mathfrak{R}^{q}$ is the basic function vector. The basic function is selected as

$$
h_{v, j}=\exp \left(\frac{-\left\|z_{2}^{T}-c_{v, j}\right\|^{2}}{\sigma_{v, j}^{2}}\right),
$$

where $c_{v, j}=\left[c_{v, j, 1}, c_{v, j, 2,}, \ldots, c_{v, j, 6}\right] \in \mathfrak{R}^{6}$ is the center value of the Gaussian function, $\sigma_{v, j}$ is the ith hidden layer node, and $j=1,2, \ldots, m . \Re^{n}$ denotes $n$-dimensional Euclidean space.

However, an ideal neural network does not exist, and the velocity vector is unknown; thus, the following neural network is used for estimation:

$$
\hat{f}_{v}=\widehat{W}_{v}^{T} h_{v}\left(z_{2}\right),
$$

where $\widehat{W}_{v}$ is the estimated value of $W_{v}^{*}$.

In accordance with the position information of the HST and the observed speed information, the tracking output feedback control law of the HST is designed as

$$
u(t)=\widehat{W}_{v}^{T} h_{v}\left(z_{2}\right)+k_{5} e_{v}+D_{2},
$$

where $k_{5}$ is a positive designed parameter and $D_{2}$ is a robust term provided in the subsequent analysis. law:

The neutral network weights use the following updating

$$
\dot{\hat{W}}_{v}=\Gamma_{v}\left(-h\left(z_{2}\right) e_{v}+\sigma_{v}\left(\widehat{W}_{v}-W_{v 0}\right)\right),
$$

where $\Gamma_{v} \in \mathfrak{R}^{q \times q}$ and is positive and $\sigma_{v}$ is a positive designed parameter.

Substituting (28), (30), and (31) into (25), we can obtain the closed-loop dynamic equations as

$$
\frac{M}{\chi} \dot{e}_{v}=-k_{5} e_{v}+\widetilde{W}_{v}^{T} h_{v}\left(z_{2}\right)+\varepsilon_{v}\left(z_{2}\right)-\frac{M}{\chi} d(t)-D_{2},
$$

where $\widetilde{W}_{v}=\widehat{W}_{v}-W_{v}^{*}$ is the estimated error.
The error boundary $\theta=\varepsilon_{v}\left(z_{2}\right)-(M / \chi) d(t)$ is defined; it is composed of neural network approximation error and external environmental disturbance. The robust term $D_{2}$ can be given as

$$
D_{2}=\gamma\left(e_{v}\right) \hat{\theta}
$$

Similar to the observer, the $\sigma^{-}$correction [38] is used to design the adaptive update law of $\widehat{\theta}$ as

$$
\dot{\hat{\theta}}=\alpha_{2}\left(\gamma\left(e_{v}\right) e_{v}+\beta_{2}\left(\widehat{\theta}-t \theta_{0}\right)\right),
$$

where the function $\gamma\left(e_{v}\right)=\tanh \left(e_{v} / \kappa_{2}\right)$ and $\kappa_{2}$ is a positive designed parameter; $\alpha_{2}$ and $\beta_{2}$ are positive designed parameters; and $\widehat{\theta}$ is an estimate of $\theta$.

Remark 3. If we select the controller (31) and the update adaptive law as (32) and (35), then the estimation error $\left(e_{s}, e_{v}\right)$ can be set to approximately zero.

\section{Stability Analysis}

Theorem 1. The nonlinear model of the HST (9) and adaptive neutral network (12) are considered. Under Assumptions 1-5, if we select the neutral network weight updating law (19) and the robust terms with its law (20), then all the signals in the closed-loop systems (13) and (14) are uniformly ultimately bounded.

Proof of Theorem 1. Considering the Lyapunov function

$$
V_{1}=\frac{1}{2} \widetilde{s}^{2}+\frac{1}{2} \widetilde{v}^{2}+\frac{1}{2} \widetilde{W}_{f}^{T} \Gamma_{f}^{-1} \widetilde{W}_{f}+\frac{1}{2 \alpha_{1}} \widetilde{\delta}^{2},
$$

and derivative $V_{1}$ provides

$$
\dot{V}_{1}=\dot{\widetilde{s}}+\tilde{\widetilde{v}}+\widetilde{W}_{f}^{T} \Gamma_{f}^{-1} \dot{\tilde{W}}_{f}+\frac{1}{\alpha_{1}} \tilde{\delta} \dot{\bar{\delta}}
$$

We select $\widetilde{W}_{f}=\widehat{W}_{f}-W_{f}^{*}$ and $\widetilde{\delta}=\widehat{\delta}-\delta$ in accordance with Assumptions 3 and 4 and the definition of $\delta$; therefore, equation (36) can be written as

$$
\dot{V}_{1}=\dot{\widetilde{s}}+\dot{\widetilde{v}} \dot{\vec{v}}+\widetilde{W}_{f}^{\mathrm{T}} \Gamma_{f}^{-1} \dot{\hat{W}}_{f}+\frac{1}{\alpha_{1}} \tilde{\delta} \dot{\bar{\delta}}
$$

Substituting (13), (18), (19), and (21) into (38), we state the dynamic equations of $V_{1}$ as 


$$
\begin{aligned}
\dot{V}_{1}= & \widetilde{s}\left(\widetilde{v}-k_{1} \widetilde{s}\right)+\widetilde{v}\left(-k_{3} \widetilde{v}-\left(k_{2}-k_{1} k_{3}\right) \widetilde{s}\right. \\
& \left.+\widetilde{W}_{f}^{T} h_{f}\left(\widehat{z}_{1}\right)-\gamma\left(\dot{\vec{s}}+k_{1} \widetilde{s}\right) \widehat{\delta}+\delta\right) \\
& +\widetilde{W}_{f}^{T} \Gamma_{f}^{-1} \Gamma_{f}\left(-h_{f}\left(\widehat{z}_{1}\right)\left(\dot{\tilde{s}}+k_{1} \widetilde{s}\right)+\sigma_{f}\left(\widehat{W}_{f}-W_{f 0}\right)\right) \\
& +\frac{1}{\alpha_{1}} \widetilde{\delta} \alpha_{1}\left(\gamma\left(\dot{\tilde{s}}+k_{1} \widetilde{s}\right)\left(\dot{\tilde{s}}+k_{1} \widetilde{s}\right)+\beta_{1}\left(\widehat{\delta}-t \delta_{0}\right)\right) \\
= & -k_{1} \widetilde{s}^{2}-k_{3} \widetilde{v}^{2}+\left(1-k_{2}+k_{1} k_{3}\right) \widetilde{s} \widetilde{s}+\widetilde{v}(-\gamma(\widetilde{v}) \delta+\delta) \\
& +\sigma_{f} \widetilde{W}_{f}^{T}\left(\widehat{W}_{f}-W_{f 0}\right)+\widetilde{\delta} \beta_{1}\left(\widehat{\delta}-t \delta_{0}\right) .
\end{aligned}
$$

Define

$$
-k_{2}+k_{1} k_{3}+1=0
$$

Lemma 1 (see [37]). $\forall \theta \in \mathfrak{R}^{n}$, and the following exists:

$$
\begin{aligned}
\tilde{\theta}^{T}\left(\widehat{\theta}-t \theta_{0}\right) & =-\|\widetilde{\theta}\|^{2}+\widetilde{\theta}^{T}\left(\theta-\theta_{0}\right) \\
& \leq-0.5\|\widetilde{\theta}\|^{2}+0.5\left\|\theta-\theta_{0}\right\|^{2} .
\end{aligned}
$$

Lemma 2 (see [34]). The inequality $h\|x\|-x^{T} h \tanh (v h x /$ $\kappa) \leq h \delta$ holds for any $\delta>0$, for any $\forall x \in \mathfrak{R}^{n}$, and $h \in \mathfrak{R}$, where $v$ is a constant that satisfies $v=e^{-(v+1)}$, that is, $v=0.2785$.

According to Lemmas 1 and 2, we obtain some inequalities as

$$
\begin{aligned}
& \int \widetilde{v} \delta-\widetilde{v} \gamma(\widetilde{v}) \delta \leq 0.2785 \kappa_{1} \delta \\
& \sigma_{f} \tilde{W}_{f}^{T}\left(\widehat{W}_{f}-W_{f 0}\right)=\sigma_{f}\left(-\left\|\tilde{W}_{f}\right\|^{2}+\tilde{W}_{f}^{\mathrm{T}}\left(W_{f}-W_{f 0}\right)\right) \\
& \leq-\frac{\sigma_{f}}{2}\left\|\widetilde{W}_{f}\right\|^{2}+\frac{\sigma_{f}}{2}\left\|W_{f}-W_{f 0}\right\|^{2} \\
& \beta_{1} \widetilde{\delta}\left(\widehat{\delta}-t \delta_{0}\right)=-\beta_{1}\|\widetilde{\delta}\|^{2}+\beta_{1} \widetilde{\delta}\left(\widehat{\delta}-t \delta_{0}\right) \\
& \leq-\frac{\beta_{1}}{2} \widetilde{\delta}^{2}+\frac{\beta_{1}}{2}\left(\widehat{\delta}-t \delta_{0}\right)^{2} \\
& \beta_{1} \widetilde{\delta}\left(\widehat{\delta}-t \delta_{0}\right)=-\beta_{1}\|\widetilde{\delta}\|^{2}+\beta_{1} \widetilde{\delta}\left(\widehat{\delta}-t \delta_{0}\right) \\
& \leq-\frac{\beta_{1}}{2} \widetilde{\delta}^{2}+\frac{\beta_{1}}{2}\left(\widehat{\delta}-t \delta_{0}\right)^{2}
\end{aligned}
$$

Then, equation (39) can be written as

$$
\begin{aligned}
\dot{V}_{1} \leq & -k_{1} \widetilde{s}^{2}-k_{3} \widetilde{v}^{2}-\frac{\sigma_{f}}{2}\left\|\widetilde{W}_{f}\right\|^{2}+\frac{\sigma_{f}}{2}\left\|W_{f}-W_{f 0}\right\|^{2} \\
& -\frac{\beta_{1}}{2} \widetilde{\delta}^{2}+\frac{\beta_{1}}{2}\left(\widehat{\delta}-t \delta_{0}\right)^{2}+0.2785 \kappa_{1}|\delta| .
\end{aligned}
$$

Let

$$
\left\{\begin{array}{l}
\mu_{1}:=\frac{\sigma_{f}}{2}\left\|W_{f}-W_{f 0}\right\|^{2}+\frac{\beta_{1}}{2}\left(\widehat{\delta}-t \delta_{0}\right)^{2}+0.2785 \kappa_{1}|\delta|, \\
\rho_{1}:=\min \left\{2 k_{1}, 2 k_{3}, \frac{\sigma_{f}}{\lambda_{\min }\left(\Gamma_{u}^{-1}\right)}, \frac{\beta_{1}}{\alpha_{1}}\right\} .
\end{array}\right.
$$

Substituting (44) into (43), we can obtain

$$
\dot{V}_{1} \leq-\rho_{1} V_{1}+\mu_{1}
$$

If $V_{1}=\Theta_{1}$ and $\rho_{1}>\left(\mu_{1} / \Theta_{1}\right)$, then $\dot{V}_{1}(t) \leq 0$.

Thus, $V_{1}(t) \leq \Theta_{1}, \forall t \geq 0$ for $V_{1}(0) \leq \Theta_{1}$.

Multiplying equation (43) by $e^{\rho_{1} t}$ provides

$$
\frac{\mathrm{d}}{\mathrm{d} t}\left(V_{1}(t) e^{\rho_{1} t}\right) \leq e^{\rho_{1} t} \mu_{1} .
$$

Defining $\varphi_{1}=\left(\mu_{1} / \rho_{1}\right)$ and integrating $(46)$ over $[0, t]$ obtains

$$
0 \leq V_{1}(t) \leq \varphi_{1}+\left(V_{1}(0)-\varphi_{1}\right) e^{-\rho_{1} t}, \quad \forall t>0 .
$$

Equation (47) shows that $V_{1}(t)$ is bounded because increasing the value of $\rho_{1}$ can be decreased. Thus, the exponential convergence of sliding surfaces is ensured. Hence, all the closed-loop control signals are uniformly ultimately bounded (UUB).

Using equation (47), we obtain the Euclidean norm as

$$
\left\|\widetilde{s}, \widetilde{v}, \sqrt{\widetilde{W}_{f}^{T} \Gamma_{f}^{-1} \tilde{W}_{f}}, \frac{1}{\alpha_{1}} \widetilde{\delta}\right\| \leq \sqrt{2 \varphi_{1}+2\left(V_{1}(0)-\varphi_{1}\right) e^{-\rho_{1} t}} .
$$

Therefore, the error variable $\widetilde{s}, \widetilde{v}, \sqrt{\widetilde{W}_{f}^{T} \Gamma_{f}^{-1} \widetilde{W}}, \widetilde{\delta} / \alpha_{1}$ can be arbitrarily decreased to $t \longrightarrow \infty$.

Theorem 2. The general HST model, which is given by equation (9), is considered. Under the conditions of Assumptions 1-5, if the control law is as (31), the neutral network weight updating laws are as (19) and (32), the robust terms law is as (34), and the adaptive update law is as (35); then, all the signals in the closed-loop systems (33) are uniformly ultimately bounded.

Proof of Theorem 2. The Lyapunov function is considered as follows:

$$
V_{2}=V_{1}+\frac{M}{2 \chi} e_{v}^{2}+\frac{1}{2} \widetilde{W}_{v}^{T} \Gamma_{v}^{-1} \tilde{W}_{v}+\frac{1}{2 \alpha_{2}} \tilde{\theta}^{2} .
$$

Derivative $V_{2}$ provides the following:

$$
\dot{V}_{2}=\dot{V}_{1}+\frac{M}{\chi} e_{v} \dot{e}_{v}+\tilde{W}_{v}^{T} \Gamma_{v}^{-1} \dot{\tilde{W}}_{v}+\frac{1}{\alpha_{2}} \tilde{\theta} \dot{\tilde{\theta}}
$$


We select $\widetilde{W}_{v}=\widehat{W}_{v}-W_{v}^{*}$ and $\tilde{\theta}=\widehat{\theta}-\theta$ in accordance with Assumptions 3 and 4 and the definition of $\theta$; thus, equation (50) can be written as

$$
\dot{V}_{2}=\frac{M}{\chi} e_{v} \dot{e}_{v}+\widetilde{W}_{v}^{\mathrm{T}} \Gamma_{v}^{-1} \dot{\hat{W}}_{v}+\frac{1}{\alpha_{2}} \tilde{\theta} \dot{\hat{\theta}} .
$$

Substituting (26), (32), and (35), into (51), we obtain the dynamic equations of $V_{1}$ as

$$
\begin{aligned}
\dot{V}_{2}= & \dot{V}_{1}+e_{v}\left(-k_{5} e_{v}+\widetilde{W}_{v}^{T} h_{v}\left(z_{2}\right)+\varepsilon_{v}\left(z_{2}\right)-\frac{M}{\chi} d(t)-D_{2}\right) \\
& +\widetilde{W}_{v}^{T} \Gamma_{v}^{-1} \Gamma_{v}\left(-h_{v}\left(z_{2}\right) z_{2}+\sigma_{v}\left(\widehat{W}_{v}-W_{v 0}\right)\right) \\
& +\frac{1}{\alpha_{2}} \tilde{\theta}\left(\alpha_{2}\left(\gamma\left(e_{v}\right) e_{v}+\beta_{2}\left(\widehat{\theta}-t \theta_{0}\right)\right)\right) .
\end{aligned}
$$

Define $\quad \theta=\varepsilon_{v}\left(z_{2}\right)-(M / \chi) d(t), \quad D_{2}=\gamma\left(e_{v}\right) \widehat{\theta}, \quad$ and $\widetilde{\theta}=\widehat{\theta}-\theta$.

Substituting $\theta, D_{2}, \widetilde{\theta}$ and equation (44) into equation (51), we can obtain

$$
\begin{aligned}
\dot{V}_{2}= & \dot{V}_{1}-k_{5} e_{v}^{2}+e_{v}\left(\theta-\gamma\left(e_{v}\right) \theta\right) \\
& +\widetilde{W}_{v}^{T} \sigma_{v}\left(\widehat{W}_{v}-W_{v 0}\right)+\widetilde{\theta} \beta_{2}\left(\widehat{\theta}-t \theta_{0}\right) .
\end{aligned}
$$

According to Lemmas 1 and 2, we obtain some inequalities as

$$
\left\{\begin{array}{l}
e_{v} \theta-e_{v} \gamma\left(e_{v}\right) \theta \leq 0.2785 \kappa_{2} \theta, \\
\tilde{W}_{v}^{\mathrm{T}} \sigma_{v}\left(\widehat{W}_{v}-W_{v 0}\right)=-\left\|\widetilde{W}_{v}\right\|^{2}+\widetilde{W}_{v}^{\mathrm{T}}\left(W_{v}-W_{v 0}\right) \\
\leq-\frac{\sigma_{v}}{2}\left\|\widetilde{W}_{v}\right\|^{2}+\frac{\sigma_{v}}{2}\left\|W_{v}-W_{v 0}\right\|^{2}, \\
\beta_{2} \tilde{\theta}\left(\widehat{\theta}-t \theta_{0}\right)=-\beta_{2}\|\widetilde{\theta}\|^{2}+\beta_{2} \tilde{\theta}^{T}\left(\theta-\theta_{0}\right) \\
\leq-\frac{\beta_{2}}{2}\|\tilde{\theta}\|^{2}+\frac{\beta_{2}}{2}\left\|\theta-\theta_{0}\right\|^{2} .
\end{array}\right.
$$

Then, equation (53) can be written as

$$
\begin{aligned}
\dot{V}_{2} \leq & \dot{V}_{1}-k_{5} e_{v}^{2}+0.2785 \kappa_{2}|\theta|-\frac{\sigma_{v}}{2}\left\|\tilde{W}_{v}\right\|^{2} \\
& +\frac{\sigma_{v}}{2}\left\|W_{v}-W_{v 0}\right\|^{2}-\frac{\beta_{2}}{2}\|\tilde{\theta}\|^{2}+\frac{\beta_{2}}{2}\left(\widehat{\theta}-t \theta_{0}\right)^{2} \\
\leq & -k_{1} \widetilde{s}^{2}-k_{3} \widetilde{v}^{2}-\frac{\sigma_{f}}{2}\left\|\widetilde{W}_{f}\right\|^{2}-\frac{\beta_{1}}{2} \tilde{\delta}^{2}-k_{5} e_{v}^{2}-\frac{\sigma_{v}}{2}\left\|\widetilde{W}_{v}\right\|^{2} \\
& -\frac{\beta_{2}}{2}\|\widetilde{\theta}\|^{2}+\frac{\sigma_{f}}{2}\left\|W_{f}-W_{f 0}\right\|^{2}+\frac{\beta_{1}}{2}\left(\widehat{\delta}-t \delta_{0}\right)^{2} \\
& +0.2785 \kappa_{1}|\delta|+\frac{\beta_{1}}{2}\left(\widehat{\delta}-t \delta_{0}\right)^{2}+0.2785 \kappa_{2}|\theta| \\
& +\frac{\sigma_{v}}{2}\left\|W_{v}-W_{v 0}\right\|^{2}+\frac{\beta_{2}}{2}\left\|\theta-\theta_{0}\right\|^{2} .
\end{aligned}
$$

Let

$$
\left\{\begin{aligned}
& \mu:=\frac{\sigma_{f}}{2}\left\|W_{f}-W_{f 0}\right\|^{2}+\frac{\beta_{1}}{2}\left(\widehat{\delta}-t \delta_{0}\right)^{2}+\frac{\beta_{2}}{2}\left(\widehat{\theta}-t \theta_{0}\right)^{2} \\
&+\frac{\sigma_{v}}{2}\left\|W_{v}-W_{v 0}\right\|^{2}+0.2785\left(\kappa_{1}|\delta|+\kappa_{2}|\theta|\right) \\
& \rho:=\min \left\{2 k_{1}, 2 k_{3}, \frac{2 \chi k_{5}}{M}, \frac{\sigma_{f}}{\lambda_{\min }\left(\Gamma_{u}^{-1}\right)}\right. \\
&\left.\alpha_{1} \beta_{1}, \frac{\sigma_{v}}{\lambda_{\min }\left(\Gamma_{v}^{-1}\right)}, \alpha_{2} \beta_{2}\right\} .
\end{aligned}\right.
$$

Substituting (56) into (55), we can obtain the following:

$$
\dot{V}_{2} \leq-\rho_{2} V_{2}+\mu_{2} \text {. }
$$

If $V_{2}=\Theta_{2}$ and $\rho_{2}>\left(\mu_{2} / \Theta_{2}\right)$, then $\dot{V}_{2}(t) \leq 0$.

Thus, $V_{2}(t) \leq \Theta_{2}, \forall t \geq 0$ for $V_{2}(0) \leq \Theta_{2}$.

Multiplying equation (57) by $e^{\rho_{2} t}$ provides

$$
\frac{\mathrm{d}}{\mathrm{d} t}\left(V_{2}(t) e^{\rho_{2} t}\right) \leq e^{\rho_{2} t} \mu_{2}
$$

Defining $\varphi_{2}=\left(\mu_{2} / \rho_{2}\right)$ and integrating (58) over $[0, t]$,

$$
0 \leq V_{2}(t) \leq \varphi_{2}+\left(V_{2}(0)-\varphi_{2}\right) e^{-\rho_{2} t}, \quad \forall t>0 .
$$

Equation (59) shows that $V_{1}(t)$ is bounded because the value of $\rho_{1}$ can be decreased. As a result, the exponential convergence of sliding surfaces is ensured. Hence, all the closed-loop control signals are UUB.

Using equation (59), we obtain the Euclidean norm as

$$
\begin{aligned}
& \left\|\widetilde{s}, \widetilde{v}, \sqrt{\widetilde{W}_{f}^{T} \Gamma_{f}^{-1} \tilde{W}_{f}}, \frac{1}{\alpha_{1}} \widetilde{\delta}, \sqrt{\frac{M}{\chi}} e_{v}, \sqrt{\widetilde{W}_{v}^{T} \Gamma_{v}^{-1} \tilde{W}_{v}}, \frac{\tilde{\theta}}{\alpha_{2}}\right\| \\
& \quad \leq \sqrt{\varphi_{2}+\left(V_{2}(0)-\varphi_{2}\right) e^{-\rho_{2} t}} .
\end{aligned}
$$

Therefore, the error variable $\left[\widetilde{\mathcal{s}}, \widetilde{v}, \sqrt{\widetilde{W}_{f}^{T} \Gamma_{f}^{-1} \widetilde{W}_{f}}\right.$, $\left.1 / \alpha_{1} \widetilde{\delta}, \sqrt{M / \chi} e_{v}, \sqrt{\tilde{W}_{v}^{T} \Gamma_{v}^{-1} \tilde{W}_{v}}, \tilde{\theta} / \alpha_{2}\right]$ can be made arbitrarily small as $t \longrightarrow \infty$ (Figure 2).

\section{Simulation and Results}

In this section, simulation studies are conducted for the designed neural network observer and the sliding mode neural network controller.

The CHR380AL HST operation control environment is simulated in MATLAB (R2017b) software. The total nominal mass of the train is $M=890$ tons, the continuous operation time is $350 \mathrm{~km} / \mathrm{h}$, the maximum operation speed is $380 \mathrm{~km} / \mathrm{h}, \chi=0.9$, and the basic general resistance is obtained as [37]

$$
f_{b}^{\prime}=0.53+0.00388 v+0.000114 v^{2} .
$$

Furthermore, the general resistance can be written as 


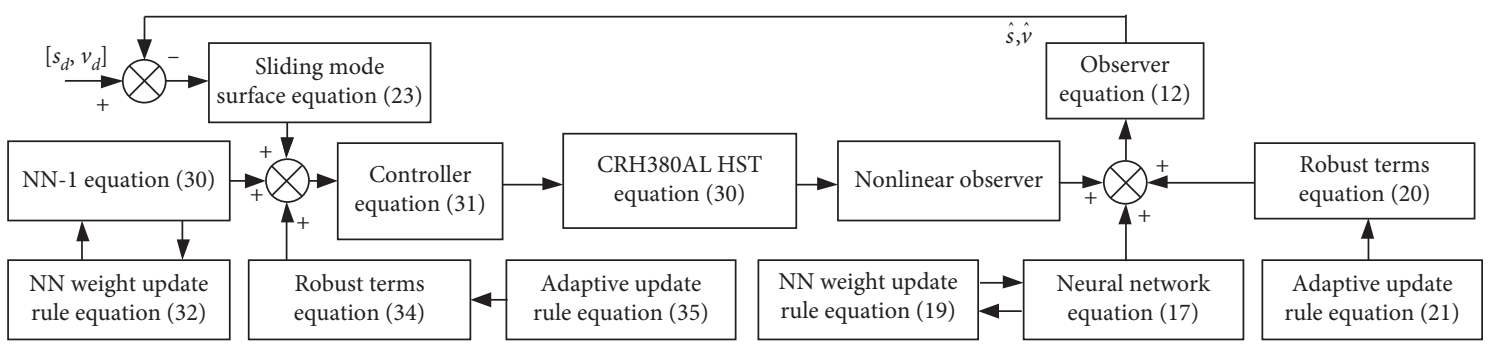

FIGURE 2: Block diagram of the overall closed-loop HST tracking system. NN: neural network; HST: high-speed train.

$$
\begin{aligned}
f_{b} & =f_{b}^{\prime} \times M \times g, \\
& \left\{\begin{array}{l}
a_{0}=4717+471.7 \sin (0.00047 t), \\
a_{1}=61.5+6.15 \sin (0.00047 t), \\
a_{2}=1.05+0.105 \sin (0.00047 t) .
\end{array}\right.
\end{aligned}
$$

The selected railway track is $561.37 \mathrm{~km}$, with one ramp, one curve, and one tunnel length. The actual operation environment conditions of the train in the computer simulation are shown in Table 1 . Such conditions lead to the changes of the additional resistance coefficients in the travel, as shown in Figure 3. Then, the two limited speed ranges are shown in Figure 4 [37]. The steps of additional resistance are calculated as follows.

(1) When $t \in[0,790.7)(s), t \in[1195.2,1284.5)(s)$, and $t \in[1500.4,2000)(\mathrm{s})$, the corresponding position is $s \in\left[0,6.83 \times 10^{4}\right)(\mathrm{m}), \quad s \in\left[10.08 \times 10^{4}, 10.83 \times 10^{4}\right)$ $(\mathrm{m})$, and $s \in\left[12.75 \times 10^{4}, 15.60 \times 10^{4}\right)(\mathrm{m})$. The general resistance coefficients are selected as (61), and the additional resistance $f_{a}=0$.

(2) For $t \in[790.7,1195.2)(\mathrm{s})$, the corresponding position is $s \in\left[6.83 \times 10^{4}, 10.08 \times 10^{4}\right)(\mathrm{m})$. The general resistance coefficients are selected as (61), and the additional resistance coefficients are selected, as shown in Table 1. Thus, equation (5) can be written as

$$
\begin{aligned}
f_{a}(t) & =f_{s}(t)=M g \sin \alpha(t)(\mathrm{N}) \\
& =g \times \sin \alpha(t)(\mathrm{N} / \mathrm{kg}),
\end{aligned}
$$

where $g$ is the acceleration of gravity.

Substituting $g=10 \mathrm{~m} / \mathrm{s}^{2}$ into (60), we obtain $f_{t}(t)$ as $0.0295 \mathrm{~N} / \mathrm{kg}$ (Table 1 ).

(3) For $t \in[1284.5,1500.4)(\mathrm{s})$, the corresponding position is $s \in\left[10.83 \times 10^{4}, 12.75 \times 10^{4}\right)(\mathrm{m})$. The general resistance coefficients are selected as (61), and the additional resistance coefficients are selected, as shown in Table 1 . We select the curve length as

$$
L_{r}=\frac{2 \pi R}{360} \varphi(t)
$$

where $R$ is the curve radius.

Substituting (61) into (3), we can obtain the following:

$$
\begin{aligned}
f_{c}(t) & =0.1029 \frac{360}{2 \pi R} M(t)(\mathrm{N}) \\
& =\frac{5.9}{R}(\mathrm{~N} / \mathrm{kg}) .
\end{aligned}
$$

Substituting $R=200 \mathrm{~m}$ into equation (62), we obtain $f_{c}(t)$ as $0.0295 \mathrm{~N} / \mathrm{kg}$ (Table 1 ).

No restricted ramp exists in the tunnel; thus, equation (4) can be written as

$$
\begin{aligned}
f_{t}(v, t) & =\frac{1.3}{10^{6}} L_{s} M(t)(\mathrm{N}) \\
& =\frac{1.3}{10^{6}} L_{s}(\mathrm{~N} / \mathrm{kg}) .
\end{aligned}
$$

Substituting $R=1000 \mathrm{~m}$ into equation (62), we obtain $f_{t}(t)$ as $0.0295 \mathrm{~N} / \mathrm{kg}$ (Table 1 ).

Substituting $L_{s}=1000 \mathrm{~m}$ into equation (63), we obtain $f_{t}(t)$ as $0.0013 \mathrm{~N} / \mathrm{kg}$.

Substituting $f_{s}(t)=0, f_{t}(t)=0.0013 \mathrm{~N} / \mathrm{kg}$, and $f_{c}(t)=$ $0.0295 \mathrm{~N} / \mathrm{kg}$ into equation (5), we obtain $f_{a}(t)$ as 0.0308 $\mathrm{N} / \mathrm{kg}$.

The disturbance is considered as $d(t)=$ $\left(10^{5} / M\right)(\sin (0.08 t)+\cos (0.08 t))$.

The desired trajectory is optimized by the genetic algorithm in [38], but the algorithm is optimized offline. If the unknown disturbance of the model parameters and external environment occur, then the open-loop control method causes a tracking error. Thus, we provide the method in this study. The designed parameters are given as $\sigma_{f, j}=2900 M$, $\sigma_{v, j}=900, \kappa_{1}=100, \kappa_{2}=200, \alpha_{1}=\alpha_{2}=0.01, \beta_{1}=\beta_{2}=1$, $\delta_{0}=2, \theta_{0}=2$.

$$
\begin{gathered}
c_{f}=100 \times\left[\begin{array}{rrrrrrrrrrrrr}
-3 & -2.5 & -2 & -1.5 & -1 & -0.5 & 0 & 0.5 & 1 & 1.5 & 2 & 2.5 & 3 \\
-3 & -2.5 & -2 & -1.5 & -1 & -0.5 & 0 & 0.5 & 1 & 1.5 & 2 & 2.5 & 3 \\
-3 & -2.5 & -2 & -1.5 & -1 & -0.5 & 0 & 0.5 & 1 & 1.5 & 2 & 2.5 & 3
\end{array}\right], \\
c_{v}=100 \times\left[\begin{array}{rrrrrrrrrrrrr}
-3 & -2.5 & -2 & -1.5 & -1 & -0.5 & 0 & 0.5 & 1 & 1.5 & 2 & 2.5 & 3 \\
-3 & -2.5 & -2 & -1.5 & -1 & -0.5 & 0 & 0.5 & 1 & 1.5 & 2 & 2.5 & 3 \\
-3 & -2.5 & -2 & -1.5 & -1 & -0.5 & 0 & 0.5 & 1 & 1.5 & 2 & 2.5 & 3 \\
-3 & -2.5 & -2 & -1.5 & -1 & -0.5 & 0 & 0.5 & 1 & 1.5 & 2 & 2.5 & 3
\end{array}\right] .
\end{gathered}
$$

The other designed parameters are shown in Table 2.

$k_{1}, k_{2}$, and $k_{3}$ are the observation parameters; $k_{4}$ and $k_{5}$ are the tracking control parameters. 
TABLE 1: Train operation conditions.

\begin{tabular}{|c|c|c|c|}
\hline \multirow{2}{*}{ Position $\mathrm{m}$} & \multicolumn{2}{|c|}{ Conditions } & \multirow[b]{2}{*}{ Tunnel length (m) } \\
\hline & Slope $(\%$ ) & Curve radius (m) & \\
\hline$s \in\left[6.83 \times 10^{4}, 10.08 \times 10^{4}\right)$ & 10 & - & - \\
\hline$s \in\left[10.83 \times 10^{4}, 12.75 \times 10^{4}\right)$ & - & 200 & 1000 \\
\hline
\end{tabular}

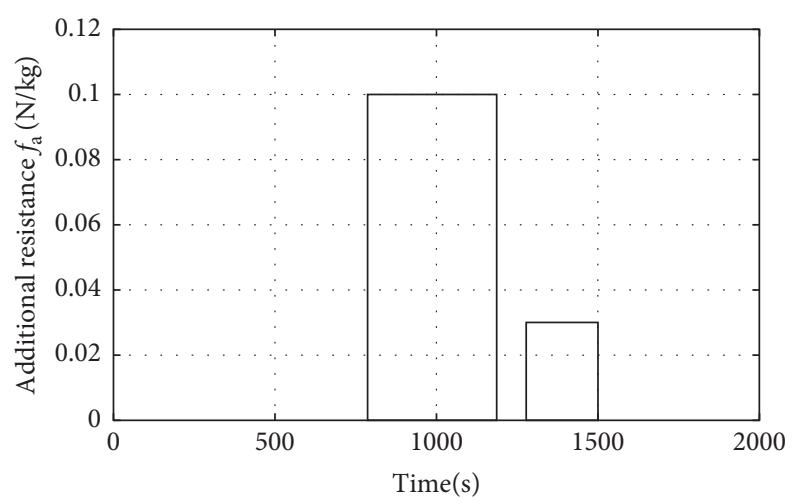

FIgURE 3: Diagram of additional resistance.

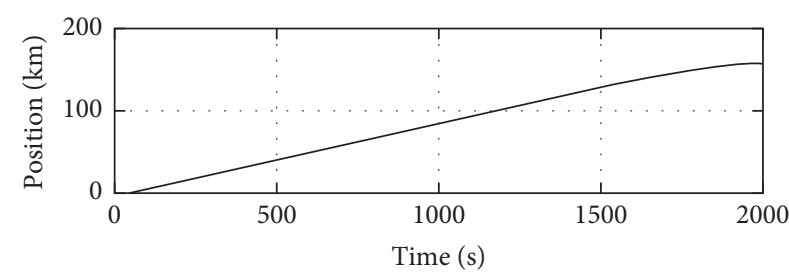

— Desired trajectory

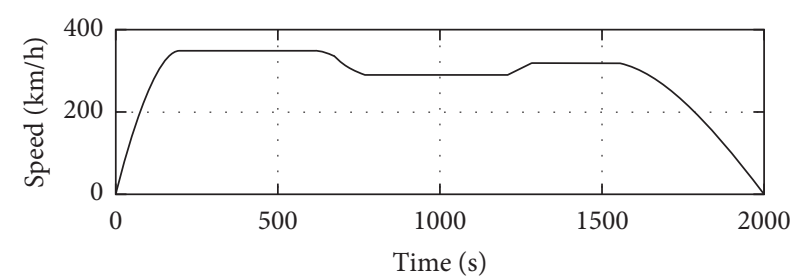

— Desired speed

(a)

(b)

Figure 4: Desired trajectory of the HST.

TABLE 2: Designed parameter variables for contrast.

\begin{tabular}{lccccccccccc}
\hline Variable & Value 1 & Value 2 & Value 3 & Value 4 & Value 5 & Value 6 & Value 7 & Value 8 & Value 9 & Value 10 \\
\hline$k_{1}$ & 0.048 & 0.06 & 0.08 & 0.4 & 0.88 & 0.88 & 4.88 & 4.88 & 8.8 & 140 \\
$k_{2}$ & 0.012 & 0.015 & 0.02 & 0.1 & 0.22 & 0.22 & 1.22 & 1.22 & 2.2 & 35 \\
$k_{3}$ & 0.06 & 0.075 & 0.1 & 0.5 & 1.1 & 1.1 & 6.1 & 6.1 & 11 & 175 \\
$k_{4}$ & 0.08 & 0.08 & 0.08 & 0.08 & 0.08 & 0.8 & 0.08 & 0.8 & 0.8 & 28 \\
$k_{5}$ & 8900 & 8900 & 8900 & 8900 & 8900 & 89000 & 8900 & 89000 & 89000 & 3115000 \\
\hline
\end{tabular}

To verify the effectiveness of the proposed algorithm and the influence of different designed parameters for HST, we propose three simulation cases, as follows:

Case 1: the control parameters are unchanged; the value of estimated parameters is increased

Case 2: the estimated parameters are unchanged; the value of control parameters is increased; all the designed parameters are increased

Case 3: the designed parameters are unchanged; the environmental disturbance is changed

In Case 1, we select the parameters from Values 1 to 5, as listed in Table 2. In Case 2, we select the parameters from
Values 5 to 9. In Case 3, we select the parameter from Value 10. Figures 5-7 are the results of Case 1. The results in Case 2 are shown in Figures $8-12$. The values in Table 3 are the energy consumption and RMS for the corresponding parameters. In Case 3, we select three types of disturbance, namely, no disturbance, fast time-varying disturbance, and slow time-varying disturbance. The parameters are as follows: $k_{1}=140, k_{2}=35, k_{3}=175, k_{4}=28$, and $k_{5}=3115000$. The energy consumption and RMS for different environmental disturbances are shown in Table 4.

As shown in Figure 5, s-s hat indicates the position observer estimation error $\widetilde{s}$; as shown in Figure $6, v-v_{\text {hat }}$ indicates the velocity observer estimation error $\widetilde{v}$. In Case 1 , the observation parameters are small. 


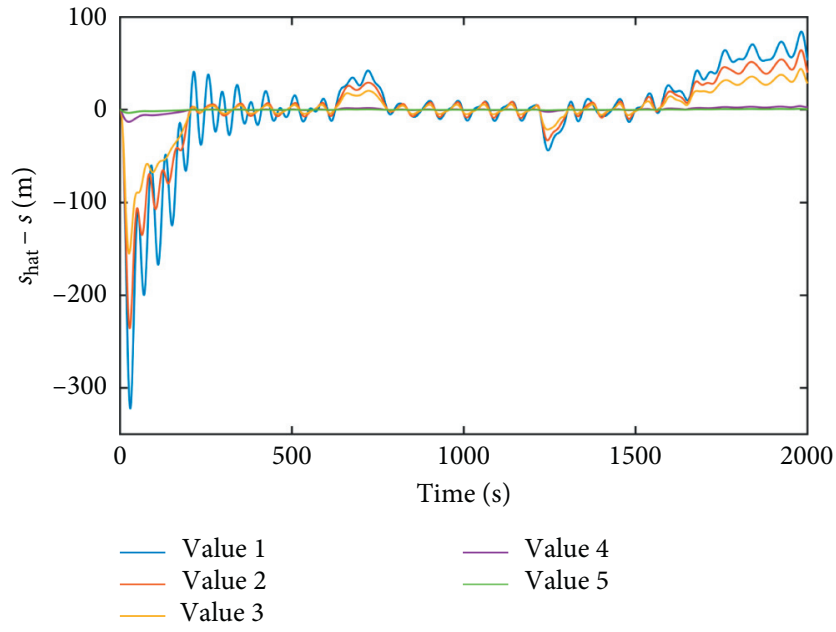

FIgURE 5: Position observer estimation error of the HST.

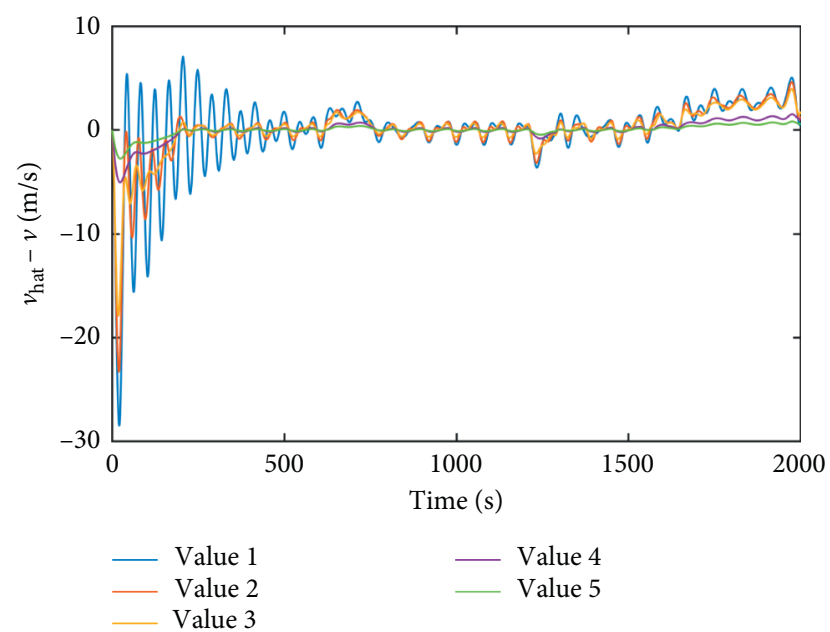

FIgURE 6: Velocity observer estimation error of the HST.

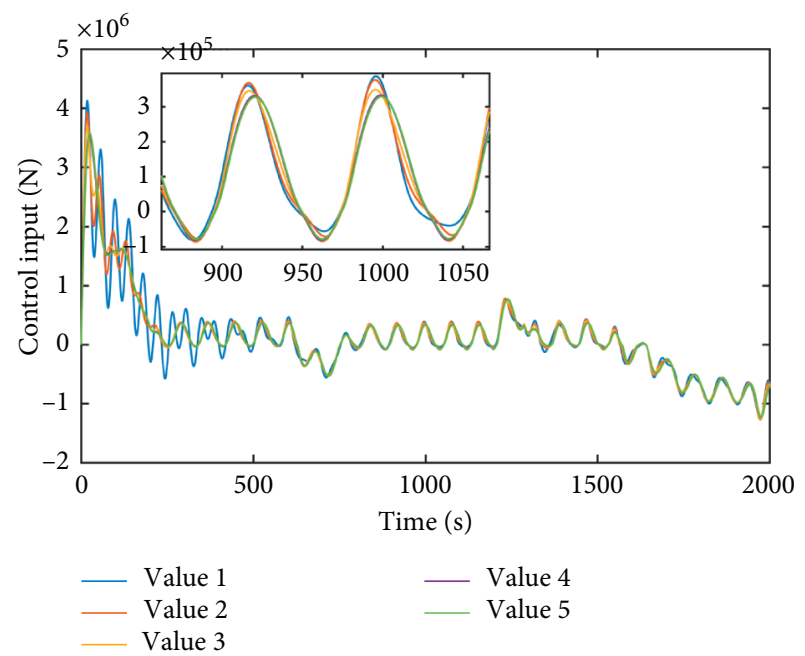

Figure 7: Control input.

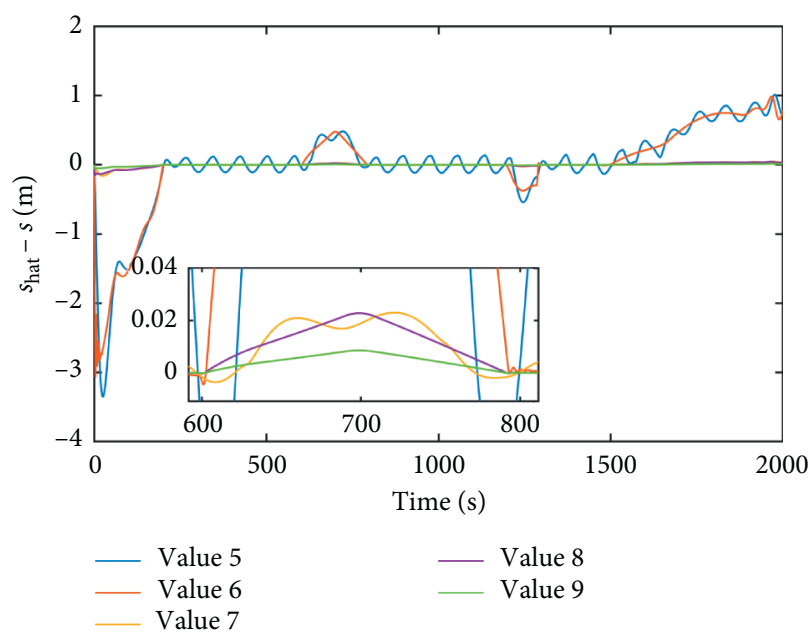

Figure 8: Position observer estimation error of the HST.

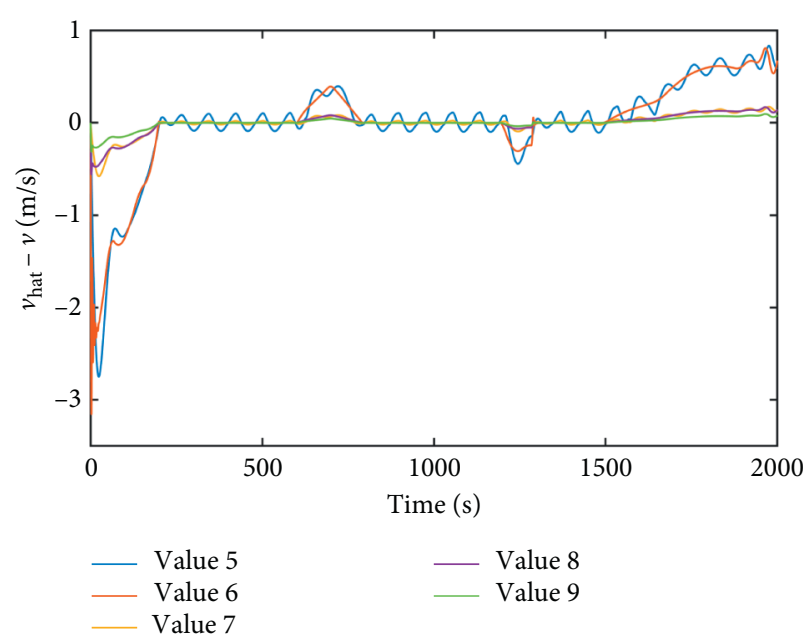

FIgURE 9: Velocity observer estimation error of the HST. 


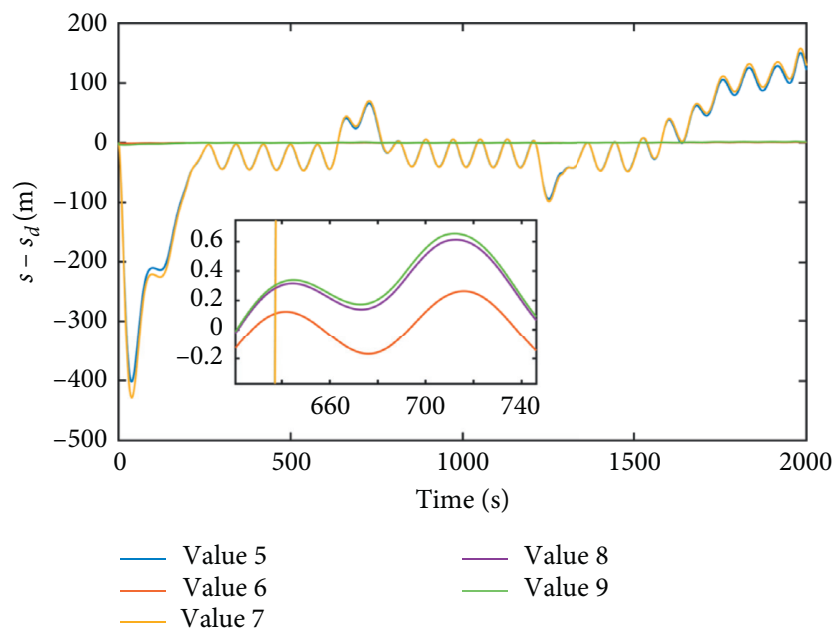

FIgURE 10: Position tracking error of the HST.

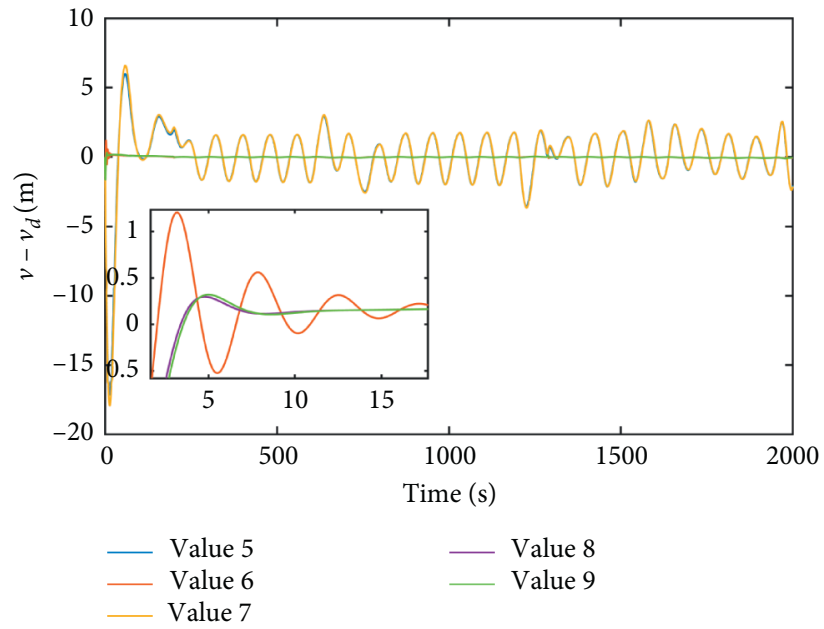

Figure 11: Velocity tracking error of the HST.

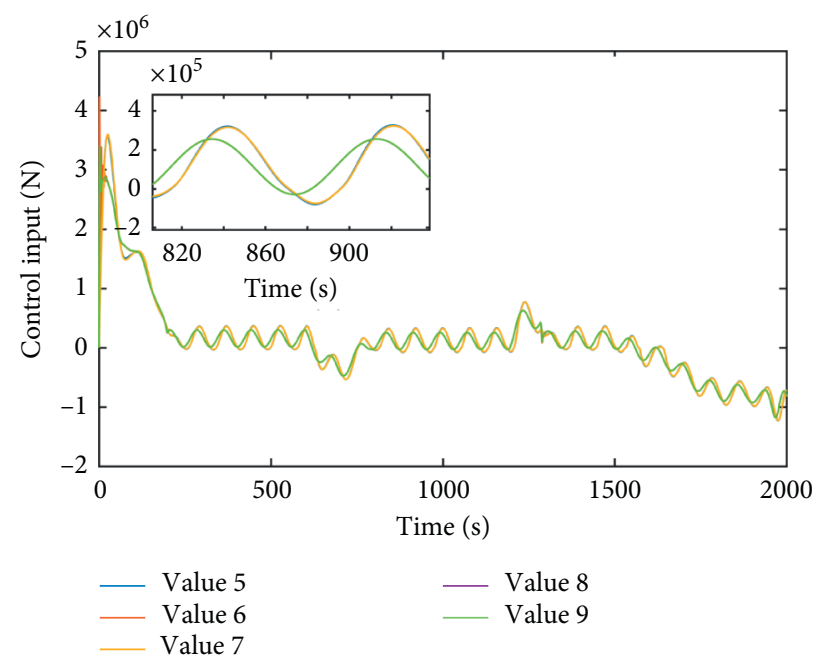

Figure 12: Control input. 
TABLE 3: Energy consumption and RMS for different parameters.

\begin{tabular}{|c|c|c|c|c|c|c|c|c|c|c|c|}
\hline \multicolumn{2}{|c|}{ Variable } & Value 1 & Value 2 & Value 3 & Value 4 & Value 5 & Value 6 & Value 7 & Value 8 & Value 9 & Value 10 \\
\hline \multicolumn{2}{|c|}{$\begin{array}{l}\text { Energy } \\
\text { consumption } \\
\left(\times 10^{11} \mathrm{~J}\right)\end{array}$} & 1 & 1.7 & 1.7555 & 37 & 1.7489 & 1. & 7438 & 1.6600 & 1.6601 & 1.6696 \\
\hline \multirow{4}{*}{ RMS } & $\widetilde{s}(\mathrm{~m})$ & 9592 & 383612 & 26.3685 & 24260 & 06257 & (2007) & 00299 & 0.0288 & 0.0108 & 0.00005 . \\
\hline & $\widetilde{v}(\mathrm{~m} / \mathrm{s})$ & 3.6695 & 2.7487 & 2.2813 & 0.9368 & 0.5132 & 0.4957 & 0.1078 & 0.1039 & 0.0585 & 0.0378 \\
\hline & $s-s_{d}$ & 65.8294 & 67.261 & 69.6498 & 84.0791 & 89.1299 & & 93.9893 & 0.8554 & & 0.0075 \\
\hline & $v-v_{d}(\mathrm{~m} / \mathrm{s})$ & 2.1981 & 1.6632 & 1.6562 & 2.0952 & 2.2006 & 0.0614 & 2.3003 & 0.06 & 0.0624 & 0.0366 \\
\hline
\end{tabular}

RMS: root mean square.

TABLE 4: Energy consumption and RMS for different environmental interferences.

\begin{tabular}{|c|c|c|c|c|}
\hline \multicolumn{2}{|l|}{ Variable } & No disturbance & Fast time-varying disturbance & Slow time-varying disturbance \\
\hline \multicolumn{2}{|c|}{ Energy consumption $\left(\times 10^{11} \mathrm{~J}\right)$} & 1.6383 & 1.6696 & 3.1443 \\
\hline \multirow{4}{*}{ RMS $\left(\times 10^{-5}\right)$} & $\tilde{s}(\mathrm{~m})$ & 5.3111 & 5.3111 & 5.3113 \\
\hline & $\widetilde{v}(\mathrm{~m} / \mathrm{s})$ & 378.4111 & 378.4073 & 378.4202 \\
\hline & $s-s_{d}(\mathrm{~m})$ & 74.997 & 75.4789 & 104.8771 \\
\hline & $v-v_{d}(\mathrm{~m} / \mathrm{s})$ & 366.2446 & 366.1317 & 366.5014 \\
\hline
\end{tabular}

In Case 1 , Value 1 is the smallest observation parameter, and Value 5 is the largest. The value of the parameters between them increases from small to large. Figures 5 and 6 show that when the observation parameters are small, the fluctuation of the position estimation error and velocity estimation error is large in the beginning. The estimation error decreases with the increase in the observation parameters. Table 3 shows that when Value 5 is selected, the RMS of the estimation error is reduced to $\widetilde{s}=0.6257$ and $\widetilde{v}=0.5132$. Energy consumption is the total energy consumption during HST operation. In Figure 7, the control input curve of Value 1 is jittery in the beginning, and the other curves almost overlap, as shown in Table 3. The energy consumptions of Values 2, 3, 4, and 5 are almost the same and smaller than that of Value 1. Therefore, the comparison of energy consumption, position estimation error, and velocity estimation error shows that a larger selection of observation parameters is better than a smaller one. The RMS value of the velocity tracking error $v-v_{d}$ is unchanged, but the RMS value of the position error $s-s_{d}$ is increased by $35.4 \%$. Therefore, the selection of parameters requires further analysis.

In Case 2, we select the designed parameters, that is, Values 5 and 6, which have the same estimated parameters and different tracking control parameters. We also consider Values 7 and 8 for comparison. Values 6,8 , and 9 have the same control parameters and different estimated parameters; this case is similar to Case 1 . In Figures 8 and 9 , the $\widetilde{s}$ and $\widetilde{v}$ curves for Values 5 and 6 almost overlap. In Table 3, the $\widetilde{s}$ RMS values of Values 5 and 6 are 0.6257 and 0.6022 , respectively. Observing the $\widetilde{v}$ curve, the RMS values of Values 5 and 6 are 0.5132 and 0.4957, respectively. In Figure 10, the position error $s-s_{d}$ of Value 5 is much larger than that of Value 6. In Figure 11, the speed tracking error $v-v_{d}$ of Value 5 is also much larger than that of Value 6. In Table 3, the $s-s_{d}$ RMS value of Value 5 is 89.1299, and the $s-s_{d}$ RMS value of Value 6 is 0.4084 . The $v-v_{d}$ RMS values of Values 5 and 6 are 2.0952 and 0.0614 , respectively.

Thus, the estimated error of $\widetilde{s}$ and $\widetilde{v}$ for Case 2 slightly improves, whereas the tracking error of $s-s_{d}$ and $v-v_{d}$ greatly improves. These conclusions can also be obtained by comparing the design parameters of Values 7 and 8. The comparison of the $s-s_{d}$ curve of Values 6, 8, and 9 in Figure 10 shows that the position tracking performance decreases slightly with the increase in the estimated parameters. However, it is also much smaller than Values 5 and 7 . The $v-v_{d}$ curve of Value 6 in Figure 11 still has a slight vibration.

In summary, increasing the value of control design parameters can reduce the tracking error of position and speed. Furthermore, increasing the value of observation estimation design parameters can reduce the speed estimation error and reduce the jitter of speed tracking error.

On the basis of the above analysis, the designed parameters of Value 10 are selected. The RMS values of $\widetilde{\mathcal{s}}, \widetilde{v}$, $s-s_{d}$, and $v-v_{d}$ in Table 3 are greatly reduced. However, as the design parameters start to increase from Value 9, energy consumption also starts to increase. The users can select parameters in accordance with their requirements.

In Case 3, we define three types of environmental disturbances to verify the robustness of the proposed algorithm, as follows:

(1) No disturbance:

$$
d(t)=0
$$

(2) Fast time-varying disturbance:

$$
d(t)=\frac{10^{5}}{M}(\sin (0.08 t)+\cos (0.08 t)) .
$$

(3) Slow time-varying disturbance:

$$
d(t)= \begin{cases}0, & t<1000(\mathrm{~s}), \\ \frac{10^{6}}{M} \sin (0.008 t), & t \geq 1000(\mathrm{~s}) .\end{cases}
$$




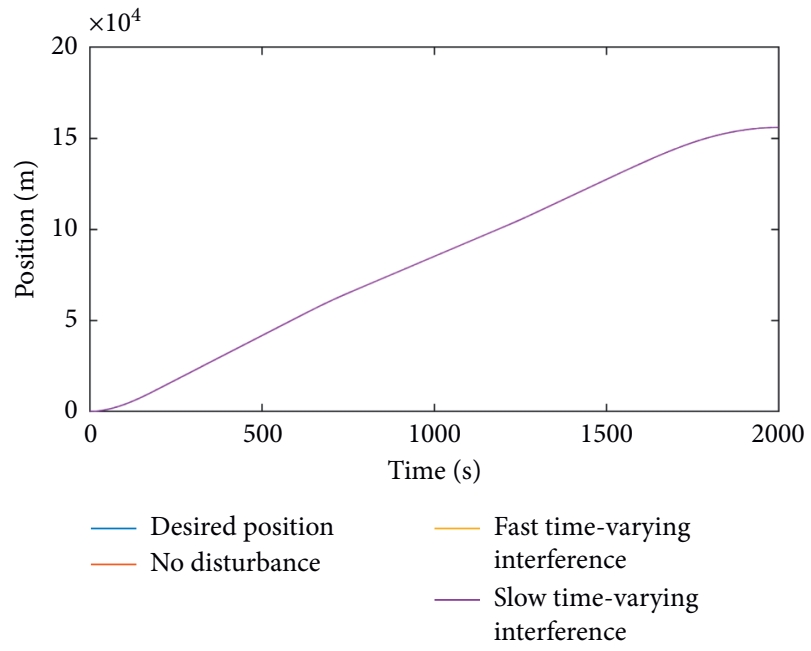

Figure 13: Position and velocity trajectory for HST.

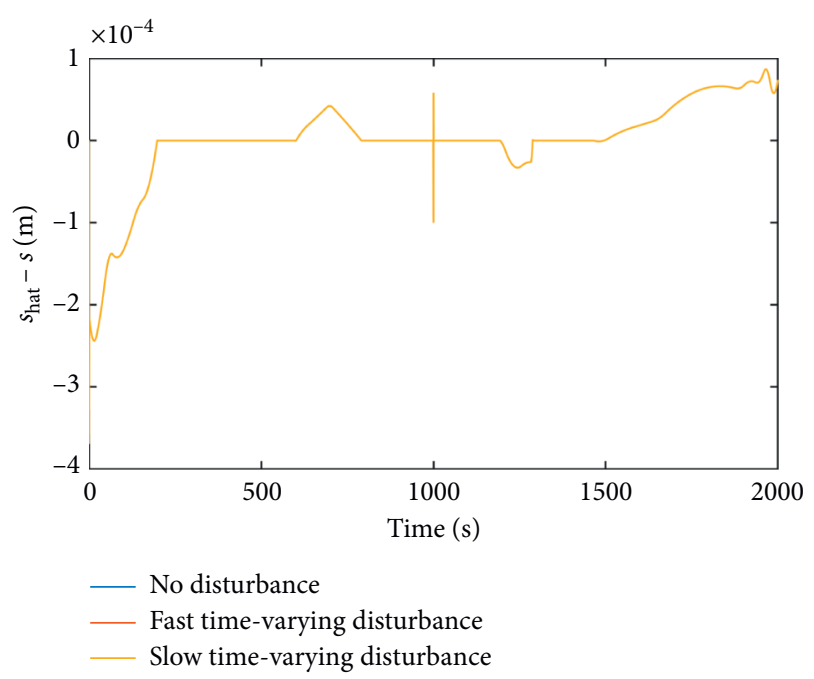

FIgURe 14: Velocity observer estimation error of the HST.

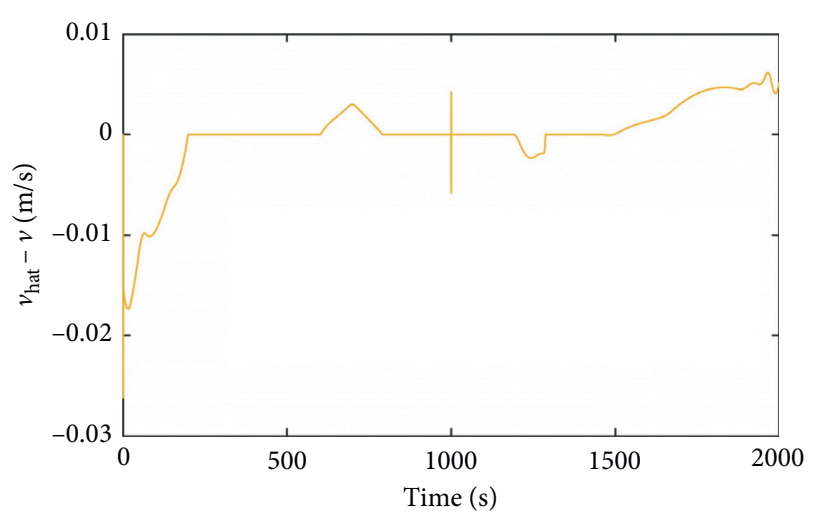

Figure 15: Velocity observer estimation error of the HST.

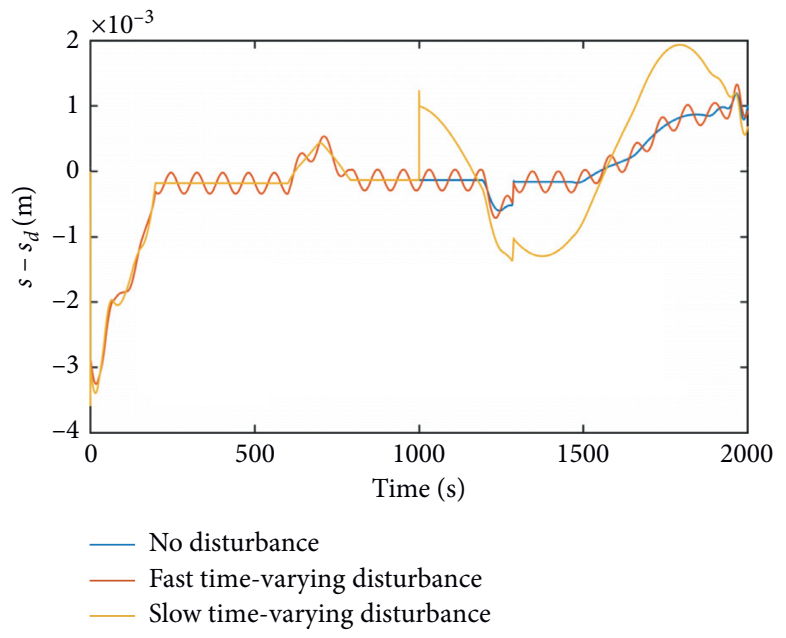

FIGURE 16: Position tracking error of the HST.

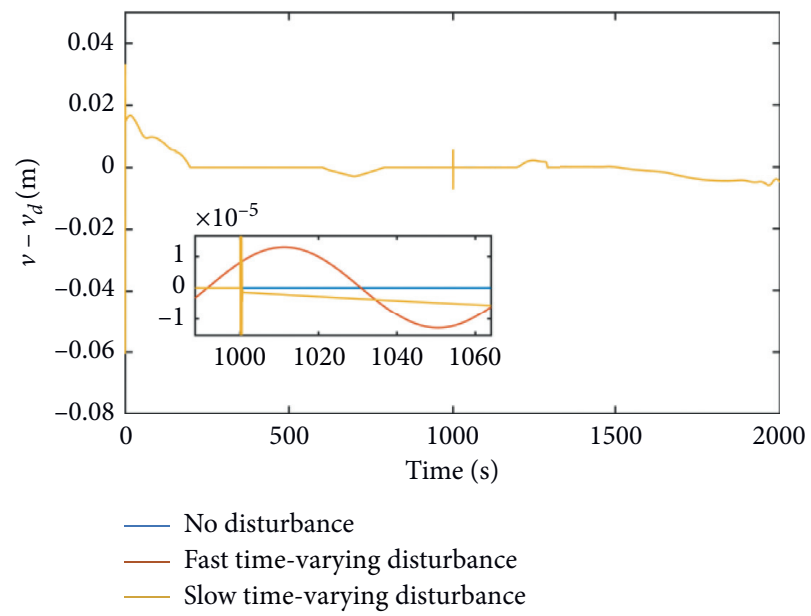

FIGURE 17: Velocity tracking error of the HST.

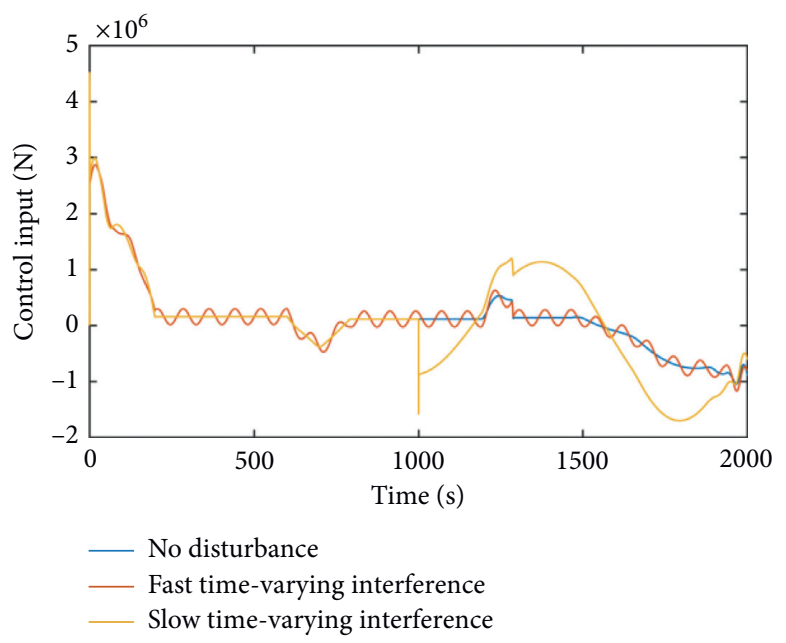

FIgURE 18: Control input with different interferences. 
Figure 13 shows the position tracking curve, and Figure 14 shows the position observer estimation error in the range of $\left[-4 \times 10^{-4}, 1 \times 10^{-4}\right](\mathrm{m})$. Figure 15 shows the speed estimation error in the range of $[-0.03,0.01](\mathrm{m} / \mathrm{s})$. Figure 16 shows the position tracking error in the range of $[-0.03,0.01](\mathrm{m} / \mathrm{s})$. Figure 17 shows the speed tracking error in the $[-0.06,0.04](\mathrm{m} / \mathrm{s})$ range. Figure 18 shows the control output in the $\left[-2 \times 10^{6}, 5 \times 10^{6}\right](\mathrm{N})$ range. Table 4 shows the RMS values of each error under three types of environmental disturbances. Table 4 shows that the RMS values increase slightly with the increase in disturbance value. As shown in Table 4, the total energy consumption reveals that HSTs need to consume relatively more energy to improve tracking performance under large disturbance.

The results show that the system can also obtain good tracking performance under large disturbance.

\section{Conclusion}

An output feedback tracking control for HSTs is designed in this study. In view of the uncertainty of the train dynamic model, the disturbance of the environment, and the immeasurable speed state, a neural network-based speed observer and a sliding mode-based speed and position tracking controller are designed. A Lyapunov-based stability analysis proves that the tracking and state estimation errors are UUB. Three simulation cases are used to test the influence of the designed parameters on speed estimation and tracking error. Therefore, adding control parameters appropriately can reduce tracking error and energy consumption. When the designed parameters are increased to a certain critical value, increasing design parameters continuously can reduce tracking error but increase the energy consumption of the control input. Conclusions from the simulation can be used as the basis of parameter selection in international operations. In future work, multiobjective optimization for energy and tracking error will be explored.

\section{Data Availability}

The data used to support the findings of this study are included within the article.

\section{Conflicts of Interest}

The authors declare that they have no conflicts of interest.

\section{Acknowledgments}

This work was supported in part by the National Natural Science Foundation (NNSF) of China under Grant 61074053.

\section{References}

[1] B. Ning, T. Tang, H. Dong et al., "An introduction to parallel control and management for high-speed railway systems," IEEE Transactions on Intelligent Transportation Systems, vol. 12, no. 4, pp. 1473-1483, 2011.
[2] P. E. Orukpe, X. Zheng, I. M. Jaimoukha, A. C. Zolotas, and R. M. Goodall, "Model predictive control based on mixed $\mathrm{H}-2 / \mathrm{H}$-infinity control approach for active vibration control of railway vehicles," Vehicle System Dynamics, vol. 46, no. 1, pp. 151-160, 2008.

[3] X.-H. Yan, B.-G. Cai, B. Ning, and W. ShangGuan, "Moving horizon optimization of dynamic trajectory planning for high-speed train operation," IEEE Transactions on Intelligent Transportation Systems, vol. 17, no. 5, pp. 1258-1270, 2016.

[4] D.-Y. Li, Y.-D. Song, and W.-C. Cai, "Neuro-adaptive faulttolerant approach for active suspension control of high-speed trains," IEEE Transactions on Intelligent Transportation Systems, vol. 16, no. 5, pp. 2446-2456, 2015.

[5] S. Sekine, N. Imasaki, and T. Endo, "Application of fuzzy neural network control to automatic train operation and tuning of its control rules," in Proceedings of the 1995 IEEE International Conference on Fuzzy Systems. The International Joint Conference of the Fourth IEEE International Conference on Fuzzy Systems and the Second International Fuzzy Engineering Symposium, pp. 1741-1746, Yokohama Japan, March 1995.

[6] G. Bing, D. Hairong, and Z. Yanxin, "Speed adjustment braking of automatic train operation system based on fuzzyPID switching control," in Proceedings of the 2009 Sixth International Conference on Fuzzy Systems and Knowledge Discovery, pp. 577-580, Tianjin, China, August 2009.

[7] H. R. Dong, B. Gao, B. Ning, and Y. X. Zhang, "Fuzzy-PID soft switch speed control of automatic train operation system," Control and Decision, vol. 25, no. 5, pp. 794-800, 2010.

[8] H. Yang, J. Yan, and K. P. Zhang, "Multiple-model self-tuning fuzzy PID control of braking process of electric multiple unit," Journal of the China Railway Society, vol. 36, no. 3, 2014.

[9] H. Yang, P. Liu, and Z.-Q. Li, "Speed tracking control for high-speed train with an Elman model," Control Theory \& Applications, vol. 34, no. 1, 2017.

[10] Z. Q. Li, H. Yang, and M. Liu, "Adaptive speed tracking control for high-speed electric multiple unit," Journal of the China Railway Society, vol. 37, no. 4, pp. 61-68, 2015.

[11] H.-r. Dong, S.-g. Gao, B. Ning, and L. Li, "Extended fuzzy logic controller for high speed train," Neural Computing and Applications, vol. 22, no. 2, pp. 321-328, 2013.

[12] C.-D. Yang and Y.-P. Sun, "Mixed $\mathrm{H}_{2} / \mathrm{H}$ cruise controller design for high speed train," International Journal of Control, vol. 74, no. 9, pp. 905-920, 2001.

[13] Z. Q. Li, J. Y. Ding, H. Yang, and J. Liu, "Generalized predictive control tuning for high-speed train based on controller matching method," Journal of the China Railway Society, vol. 40, no. 9, pp. 82-89, 2018.

[14] D. C. Li, J. J. Meng, R. X. Xu, and M. Yin, "Sliding mode adaptive robust $\mathrm{H}_{\infty}$ conrol method for high-speed train under strong wind conditions," Journal of the China Railway Society, vol. 40, no. 7, pp. 67-73, 2018.

[15] M. Faieghi, A. Jalali, and S. Kamal-e-ddin Mousavi Mashhadi, "Robust adaptive cruise control of high speed trains," ISA Transactions, vol. 53, no. 2, pp. 633-541, 2014.

[16] Z. Zhong, Y. Wang, C. Yin, and T. Tao, "Terminal iterative learning control based station stop control of a train," International Jounal of Control, vol. 84, no. 7, pp. 1263-1274, 2011.

[17] C. T. Chris and Y. Tan, "Iterative learning control with mixed constraints for point-to-point tracking," IEEE Transaction Control Systems Technology, vol. 21, no. 3, pp. 604-616, 2013.

[18] H. Ji, Z. Hou, and R. Zhang, "Adaptive iterative learning control for high-speed trains with unknown speed delays and 
input saturations," IEEE Transactions on Automation Science and Engineering, vol. 13, no. 1, pp. 260-273, 2016.

[19] Q. Yu, Z. Hou, and J.-X. Xu, "D-type ILC based dynamic modeling and norm optimal ILC for high-speed trains," IEEE Transactions on Control Systems Technology, vol. 26, no. 2, pp. 652-663, 2018.

[20] H. Yang and Y. T. Fu, "A survey of modeling and speed control methods for operation of trains," Journal of East China Jiaotong University, vol. 35, no. 5, pp. 1-8, 2018.

[21] K. Xia, H. Gao, L. Ding et al., "Trajectory tracking control of wheeled mobile manipulator based on fuzzy neural network and extended Kalman filtering," Neural Computing and Applications, vol. 30, no. 2, pp. 447-462, 2018.

[22] Z. Zhao, W. He, and S. S. Ge, "Adaptive neural network control of a fully actuated marine surface vessel with multiple output constraints," IEEE Transaction Control Systems Technology, vol. 22, no. 4, pp. 1536-1543, 2014.

[23] M. Van, "Adaptive neural integral sliding-mode control for tracking control of fully actuated uncertain surface vessels," International Journal of Robust and Nonlinear Control, vol. 29, no. 5, pp. 1537-1557, 2019.

[24] A.-M. Zou, K. D. Kumar, Z.-G. Hou, and X. Liu, "Finite-time attitude tracking control for spacecraft using terminal sliding mode and Chebyshev neural network," IEEE Transactions on Systems, Man, and Cybernetics, Part B (Cybernetics), vol. 41, no. 4, pp. 950-963, 2011.

[25] H. Khebbache and M. Tadjine, "Robust fuzzy backstepping sliding mode controller for a quadrotor unmanned aerial vehicle," Control Engineering \& Applied. Informatics.vol. 15, no. 2, 2013.

[26] J. T. Fei and C. Lu, "Adaptive sliding mode control of dynamic systems using double loop recurrent neural network structure," IEEE Transactions on Neural Networs and Learning Systems, vol. 29, no. 4, pp. 1275-1286, 2018.

[27] C. L. P. Chen, C.-E. Ren, and D. Tao, "Fuzzy observed-based adaptive consensus tracking control for second-order multiagent systems with heterogeneous nonlinear dynamics," IEEE Transactions on Fuzzy Systems, vol. 13, no. 9, pp. 906-915, 2014.

[28] B. Chen, X. Liu, and C. Lin, "Observer and adaptive fuzzy control design for nonlinear strict-feedback systems with unknown virtual control coefficients," IEEE Transactions on Fuzzy Systems, vol. 26, no. 3, pp. 1732-1743, 2018.

[29] Y. Xu, H. Liang, T. Li, and H. Ma, "Observer-based adaptive control for nonlinear strict-feedback stochastic systems with output constraints," International Journal of Robust and Nonlinear Control, vol. 29, no. 5, pp. 1515-1536, 2019.

[30] C. Cheng, Y. Zhang, and S. Liu, "Neural observer-based adaptive prescribed performance control for uncertain nonlinear systems with input saturation," Neurocomputing, vol. 370, pp. 94-103, 2019.

[31] D. Ding, Z. Wang, Q.-L. Han, and G. Wei, "Neural-networkbased output-feedback control under round-robin scheduling protocols," IEEE Transactions on Cybernetics, vol. 49, no. 6, pp. 2372-2384, 2019.

[32] K. Shojaei, "Neural adaptive output feedback formation control of type (m, s) wheeled mobile robots," IET Control Theory \& Applications, vol. 11, no. 4, pp. 504-515, 2017.

[33] H. Wang, D. Wang, and Z. H. Peng, "Adaptive neural control for cooperative path following of marine surface vehicles: state and output feedback," International Journal of Systems Science, vol. 47, no. 2, pp. 343-359, 2016.

[34] S. Z. Peng, X. L. Zhang, N. Zhang, and G. Guo, "Recursive sliding mode dynamic surface output feedback control for ship trajectory tracking based on neural network observer," Control Theory \& Applications, vol. 35, no. 8, pp. 1092-1100, 2018.

[35] F. Zouari, A. Ibeas, A. Boulkroune, J. Cao, and M. M. Arefi, "Neuro-adaptive tracking control of non-integer order systems with input nonlinearities and time-varying output constraints," Information Sciences, vol. 485, pp. 170-199, 2019.

[36] V. Garg, Dynamics of Railway Vehicle Systems, Elsevier, New York, NY, USA, 1984.

[37] J. Chen, High-Speed Train Fuzzy Tracking Contro via Ho Tracking Performace, East China Jiaotong University, Nanchang, China, 2018.

[38] Y. Liu and W. D. Li, "An ATO multi-objective optimization control strategy based on genetic algorithm," in Proceedings of the 31th Chinese Control and Decision Conference, pp. 12201224, Nanchang, China, June 2019. 